\title{
Sur la répartition spatiale des Crustacés dans le sous-écoulement d'un ruisseau des Pyrénées
}

\author{
R. Rouch'
}

Mots clés : milieu interstitiel hyporhéique, peuplement des Crustacés, Harpacticides, répartition spatiale.

Le peuplement des Crustacés du sous-écoulement du ruisseau de Lachein (Pyrénées) a été étudié sur un site de 75 m² $^{2}$ et à une profondeur de $0,60 \mathrm{~m}$.

Les écoulements de surface déterminent quatre zones principales, deux bancs de graviers, un chenal et une zone d'eau mortc. La porosité ( 20 à $30 \%$ ), plus élevée dans les bancs de graviers que dans le chenal, est également plus forte à l'amont des bancs qu’à l'aval. La granulomét rie diffère selon les zones, les bancs de graviers étant caractérisés par une plus forte abondance des éléments mesurant 1 à $8 \mathrm{~mm}$. Les caractéristiques chimiques du souséécoulement, toujours proches de celles du cours subaérien, évoluent de façon identique à ces dernières au cours des saisons. Les valeurs de l'o dissous, proches de la saturation en surface, sont comprises entre $0,02 \%$ et $84,7 \%$ dans le sous-écoulement où leur distribution dépend très directement de la dynamique des écoulements de surface. Les teneurs en matière organique particulaire, comprises entre 4 et 13,6\%, subissent une évolution saisonnière. Les valeurs moyennes les plus basses caractérisent le chenal.

Les Crustacés, représentés par 50 espèces et sous-especes, dont 22 taxons stygobies, montrent une forte similitude faunistique ave ceux colonisant le système karstique du Baget dont le ruisseau de Lachein est issu. Ils sont caractérisés par une organisation stable dans le temps, dominée par les Copépodes et, notamment, par les Harpacticides qui constituent $60 \%$ du peuplement. Leur densité moyenne est de 369 individus/10 1. La répartition spatiale des Malacostracés comme des Harpacticides est très variable, avec des especes eurytopes (formes épigées) et sténotopes (formes hypogées) ; parmi ces dernières, les unes sont inféodées aux zones les plus oxygénées, d'autres au contraire, sont limitées aux zones les moins oxygénées. Chez les Harpacticides, les densités comme les pourcentages des formes hypogées sont très dì rectement liés aux différentes zones définies par les écoulements de surface.

Spatial distribution of crustaceans in the hyporheic habitat of a Pyrenean brook.

Kuywords : hyporheic habitat, crustacean community. Harpacticoida. spatial distribution.

The crustacean assemblages of ruisseau de Lachein (Pyrenées) underflow were studied on a site of $75 \mathrm{~m}^{2}$ at a depth of $0.6 \mathrm{~m}$. Four main zones are created by the surface flow, i.e. two gravel banks, a channel and a stagnant water zone. The porosity $(20-30 \%)$ is higher in the gravel banks than in the channel. It is also higher upstream than dowstream. The grain size differs in relation to the four zones, the gravel banks possessing a larger amount of elements measuring 1 to $8 \mathrm{~mm}$.

The underflow and the brook have close chemical characteristics varying in the same manner through the season. Oxy. gen levels are close to saturation in the water surface, but are be tween $0,02 \%$ and $84,7 \%$ in the underflow where their values are directly affected by the influence of the surface flow. The proportion of organic matter (4 $13.6 \%$ ) follows a seasonal pattern, the lowest mean ratio being that of the channel.

Among the $50 \mathrm{sp}$. and sub. sp. of Crustacea in the underflow, 22 are stygobiont (hypogeous) taxa. These taxa show a strong faunistic similarity with the community of the Baget karstic system from which the ruisseau de Lachein originates. This fauna is characterized by a stable organisation throughout the entire year and is dominated by Copepoda, particularly Harpacticoida representing $60 \%$ of the population. The average density is 369 animalsi 10 I. The Malacostraca and the Harpacticoida maintain a variable spatial distribution with eurytope and stenotope species. Among stenotope species, some taxa are restricted to the region with the highest amount of $\mathrm{O}_{2}$. whereas others are limited to weakly oxygenated areas.

The different zones defined by the surface flow have a direct influence on the density as well as on the percentage of harpacticoid hypogeous forms.

1. Laboratoire souterrain du CNRS, Moulis, 09200 Saint-Girons, France. 
Les recherches sur les milieux interstitiels d'eau douce ont connu, au cours des trois dernières décennies, un développement important (voir historique in Danielopol 1982) et, notamment, celles consacrees au milieu hyporhéique (Orghidan 1959). En effet, la présence de nombreuses formes épigées dans le sous-écoulement (Angelier 1953, Schwoerbel 1961) a conduit les hydrobiologistes à reconnaitre que l'estimation réelle du benthos des cours d'eau passe par une connaissance approfondie du peuplement de leur lit sédimentaire (Williams \& Hynes 1974, Williams 1981, Bretschko \& Klemens 1986, S1anford \& Ward 1988).

Mais beaucoup de recherches en ce domaine tiennent à la richesse du sous-écoulement en formes hypogées comme l'ont démont ré Karaman dès 1936 et Chappuis (1942).

Au point de vue écologique, plusieurs niveaux d'échelle ont été retenus. Beaucoup de travaux pionniers présentent un caractère régional: Pyrénées (Angelier 1953) et Vosges (Picard 1962) en France, zone entre le Harz et la Weser (Husmann 1956), Forêt-Noire (Schwoerbel 1959) et Thuringe (Ronneberger 1975) en Allemagne, affluents du Danube (Ruttner-Kolisko 1961) et Arlberg (Tilzer 1968) en Autriche, Carpates blanches (Sterba \& Holzer 1977) en Tchécoslovaquie. Viennent ensuite des recherches à l'échelle du cours d'eau ou d'une de ses portions : Tarn (Bou 1968), Nert (Gourbault \& LescherMoutoué 1968), Rhône (Gibert et al. 1977, Dole 1983), Save (Mestrov et al. 1983), Bela (Vranovsky 1984), Adige (Ruffo 1961. Ferrarese \& Sambugar 1976), Brenta (Braioni et al. 1980), Mur (Kirchengast 1984), Danube (Danielopol 1983). A l'échelon local, enfin, apparaissent des recherches intensives poursuivies sur un site déterminé : station de Marsal sur le Tarn (Bou 1979), Ritrodat sur le Seebach (Bretschko 1981), Salem Creek dans l'Ontario (Godbout \& Hynes 1982), Eberschuttwässer sur le Danube (Dan ielopol 1983), canal de Miribel sur le Rhône (Marmonier \& Dole 1986).

C'est cette dernière approche qui a été utilisée sur le ruisseau de Lachein (Commune d'Alas-Balaguères, Ariège). Ce ruisseau draine les eaux issues du système karstique du Baget (Mangin 1974) vers le Lez, affluent de rive gauche du Salat. Plusieurs sondages (Bou \& Rouch 1967, Rouch 1968) avaient montré la richessé du sous-écoulement de ce ruisseau en formes hypogées.
Après les recherches menées sur le système karstique du Baget (Rouch 1977, 1984), il était intéressant d'analyser le peuplement d'un milieu interstitiel en liaison avec un aquifère karstique.

\section{Le site étudié}

Le ruisseau de Lachein mesure $1,3 \mathrm{~km}$ de long entre sa source (altitude $498 \mathrm{~m}$ ) et son embouchure (altitude $475 \mathrm{~m}$ ): sa pente est, en moyenne, de $1,77 \%$. Le cours d'eau, encaissé entre des rives abruptes $(1.2 \mathrm{~m})$, mesure 4 à $5 \mathrm{~m}$ de large en moyenne et ne possède aucune plage exondée, même à l'étiage.

Il n'existe qu'un affluent entre la source et l'embouchure, le ruisseau de Plamicou, dont les apports d'eau sont négligeables si bien que les débits du ruisseau sont pratiquement assurés par les eaux issues du système du Baget. Celui-ci a une superficie de $13,25 \mathrm{~km}^{2}$ pour une altitude moyenne de $923 \mathrm{~m}$; l'amont du bassin versant est exempt de toute source de pollution en raison de l'absence d'habitat permanent, de route et de culture; la valeur moyenne des précipitations y est de $1755 \mathrm{~mm} / \mathrm{an}$ (période de 1971 à 1984). Le débit moyen du ruisseau est de $0,523 \mathrm{~m}^{3 /} / \mathrm{s}$ (période de 1969 a 1984), les valeurs minimales et maximales observées étant de $9,4 \mathrm{~m}^{3 /} / \mathrm{s}$ et de $0,05 \mathrm{~m}^{3} / \mathrm{s}$. Le régime est de type pluvial océanique avec de hautes eaux hivernales et printanières et des basses eaux estivales et automnales. Excepté les périodes de crues, la hauteur de l"eau libre dans le ruisseau dépasse rarement $0.50 \mathrm{~m}$.

A $830 \mathrm{~m}$ en aval de sa source, le ruisseau s'élargit à proximité d'un seuil naturel aménagé en barrage. A cet endroit (fig. 1), une zone de $15 \mathrm{~m}$ de long sur $5 \mathrm{~m}$ (en moyenne) de large, soit une superficie de 75 $\mathrm{m}^{2}$, a été divisée de l'aval vers l'amont, en cinq bandes transversales de 3 m chacune. Ces cinq bandes, subdivisées selon la ligne médiane du cours d'eau, permettent de définir 10 secteurs désignés par les lettres A à I. Cette étude est donc limitée au sousécoulement (sensu stricto) du ruisseau.

\section{Matériel et mẹ́thodes}

Après la méthode des sondages KaramanChappuis, les techniques de prélèvement de la faune interstitielle se sont orientées vers deux voies 


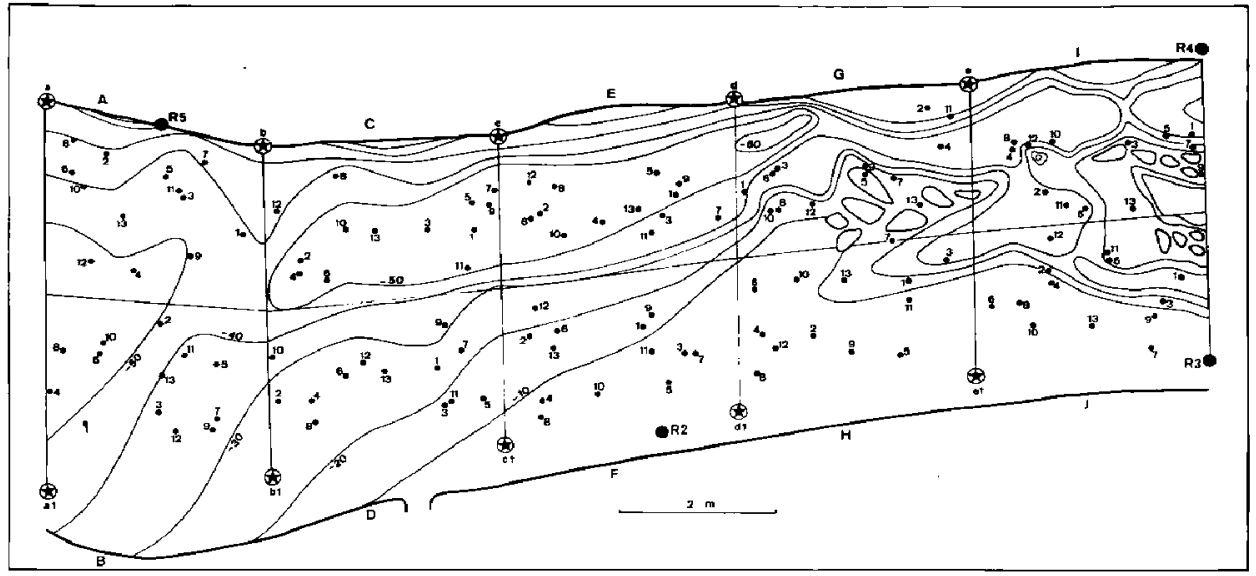

Fig. 1. Carte du site étudié : les lignes de niveau $(-10,-20$, etc.) correspondert à des profondeurs mesurées pendant l'étiage $(80 \mathrm{~J} / \mathrm{s})$. Le site a été divisé en 10 secteurs (A à J), chaque point de pompage étant repéré par ses coordonnées (A1, A2, B1, B2 etc. correspondent à des prélèvements de septembre 1985, A3, A4, B3, B4 etc. à des prélèvements de novembre 1985 et ainsi de suite).

différentes, celle des carottages et celle des pompages.

La méthode de carottage la plus simple consiste à enfoncer dans le lit du cours d'eau un tube non obstrué à sa base et à récupérer la colonne de sédiments contenue à l'intérieur du tube. Facile à mettre en ceuvre dans des sédiments fins non compactés (Whitman \& Clark 1984), cette méthode est plus difficilement applicable à des sédiment $s$ off rant une fraction grossière importante, ce qui est le plus souvent le cas. Sterba \& Holzer (1977) ont alors utilisé des tubes de fort diamètre $(142 \mathrm{~mm}$ ) ce qui endommage fortement le point de prélèvement. Cet te critique est également valable pour la technique proposée par Stocker \& Williarns (1972) qui se fonde sur la congélation des sédiments autour d'un tube servant a injecter le liquide réfrigérant; Bretschko \& Klemens (1986) font observer que, indépendarnment de son prix de revient et du temps qu'elle nécessite, cette méthode perturbe la zone d'échantillonnage pour plusieurs mois ce qui rend impossible toute série de prélèvements au même point. Autre méthode enfin, celle proposée par Williams \& Hynes
(1974) qui permet de prélever un volume précis de sédiments à la profondeur choisie. Selon Exner \& Davies (1979), elle serait limitée par la taille des ouvertures pratiquées dans la sonde $(1 \mathrm{~cm})$, l'accroissement du diamètre du tube de prélèvement (donc celle des ouvertures) multipliant les difficultés d'enfoncement.

La technique de pompage (Bou \& Rouch 1967. Husmann 1971) présente le désavantage, selon Exner \& Davies (1979), de ne pas fournir de prélèvements distincts, instantanés, à une profondeur précise.

Toutefois, Danielopol (1976) estime que les dix premiers litres pompés sont représentatifs de la communauté qui existe autour du tube sur une hauteur de $30 \mathrm{~cm}$ environ par rapport à la crépine. Un in térêt supplémentaire de cette méthode, outre sa facilité de mise en ouvre, réside dans le fait qu'elle autorise des prélèvements à intervalles réguliers sur des sondes placées à demeure (Bou 1979, Danielopol 1983). Toutefois, Gibert et al (1981) puis Dole (1983) ont souligné les modifications entrainées par des 
pompages successifs sur de telles sondes. Bretschko (1985) préconise, pour minimiser l'effet de tels pompages, d'utiliser les tubes plantés à demeure comme des pièges, les orifices de la crépine n'étant ouverts que périodiquement et seule l'eau à l'intérieur du tube étant aspirée.

L'objectif de la présente étude n'étant pas d'étudier la micro-distribution verticale des individus dans les sédiments mais davantage leur répartition sur l'ensemble du site, la méthode de pompage, assez globale, paraissait la plus appropriée en raison de sa facilité d'emploi. Afin d'éviter les modifications entraînées par des pormpages successifs sur des sondes fixes, aucun prélèvement n'a été réalisé au même endroit. Tous les deux mois, pendant un an (septembre 1985 - septembre 1986), vingt échantillons (deux par secteur), repérés par leurs coordonnées, ont été prélevés à une profondeur moyenne de $60 \mathrm{~cm}$ dans les sédiments.

La série de contrôle de septembre 1986 ne comportant que dix échantillons, ce sont 130 prélève. ments qui ont été réalisés au total. Chacun d'entre eux est caractérisé par la lettre du secteur dont il est issu et par un numéro: $1-2$ (septembre 1985) ; 3-4 (novembre 1985) ; 5-6 (février 1986) ; 7-8 (mars 1986) ; 9-10 (mai 1986) : 11-12 (juillet 1986) ; 13 (septembre 1986) (voir figure 1).

Les dix premiers lit res pompés étaient filtrés sur un filet de $100 \mu \mathrm{m}$ de maille; le résidu solide, fixé dans l'alcool à $70^{\circ}$, a servi à l'analyse faunistique où seuls les Crustacés ont été pris en compte. 2,5 litres étaient ensuite prélevés aux fins d'analyse chimique, dont deux litres pour l'étude de la matière organique particulaire et 0,5 l pour celle des ions. 0,5 I d'eau du cours subaérien était également prélevé lors de chaque séríe de pompage. La température de l'eau (thermomètre au 1/10) et l'oxygène dissous (oxymètre WTW - Aquadata AOX 100) étaient mesurés sur place.

Les Crustacés, après coloration au rose bengale. ont été triés dans leur totalité pour les quatre premières séries de prélèvement. Par la suite, les Ostracodes n'ont plus été isolés.

La porosité et la granulométrie ont été estimées à partir d'échantillons congelés par la méthode de l'azote liquide (Stocker \& Williams 1972) modifiee par Danielopol et al (1980) avec notamment l'utilisation d'un tube de diamètre interne égal à $3,5 \mathrm{~cm}$.

\section{Caractéristiques physiques}

Les sept séries de prélèvements ont été réalisées pour des débits moyens du ruisseau (fig. 2) correspondant à des valeurs d'étiage (septembre $1985=$ $0,075 \mathrm{~m}^{3 /} / \mathrm{s}$, novembre $1985=0,117 \mathrm{~m}^{3 /} / \mathrm{s}$, juillet 1986 $=0,095 \mathrm{~m}^{3 / \mathrm{s}}$, septembre $1986=0,100 \mathrm{~m}^{3 /} \mathrm{s}$ ) ou de hautes eaux (février $1986=0,427 \mathrm{~m}^{3 / \mathrm{s}}$, mars 1986 $=0,620 \mathrm{~m}^{3} / \mathrm{s}$, mai $1986=0,207 \mathrm{~m}^{3} / \mathrm{s}$ ).

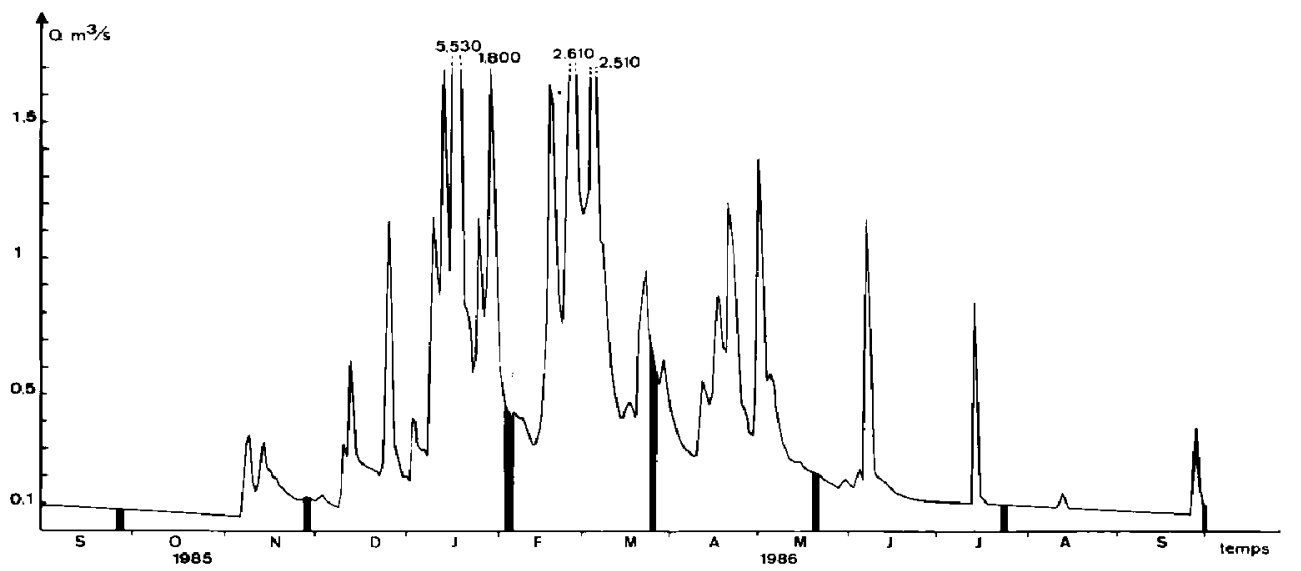

Fig. 2. Débits du ruisseau de Lachein et périodes de prélèvements. 


\subsection{Distribution des écoulements sur le site}

Six profils de vitesse ont été effectués suivant les différentes lignes transversales destinées à quadriller le site. Le débit du ruisseau était de $0,345 \mathrm{~m}^{3 /} / \mathrm{s}$ (fig. 3 a,b,c).

A l'entrée de la zone étudiée (profil R4-R3), il existe deux écoulements principaux, l'un en rive droite, l'autre en rive gauche, marqués tous les deux par des vitesses de $60 \mathrm{~cm} / \mathrm{s}$. Le flux de droite vient longer la rive qu'il affouille (profil d-dl), avant d'infléchir sa course vers le centre du cours d'eau. Le flux de rive gauche se divise à plusieurs reprises et vient ainsi alimenter le flux de rive droite avec lequel il finit par se confondre à l'aval du site étudié.

Cette distribution du courant se traduit, sur le plan topographique, par l'existence de plusieurs zones qui peuvent être schématisées de la façon suivante.

- Deux zones de dépôts : l'une, en rive gauche, recouvre les secteurs $D, F, H, J$; l'autre en rive droite, plus petite, occupe le secteur A et une partie du secteur $C$. Les vitesses d'écoulement y sont faibles.

- Une zone de creusement : elle correspond au chenal qui se développe dans les secteurs I, G, E et $C$ (en partje), Les vitesses d'écoulement $y$ sont élevées.

- Une fosse avec des eaux mortes : secteur B.

\subsection{La porosité}

Les valeurs de la porosité ont été calculées à partir de neuf échantillons congelés provenant chacun de secteurs différents (secteur D non étudié). Les sédiments formaient une chape de 30 à $40 \mathrm{~cm}$ de hauteur à partir de la base du tube, celui-ci étant enfoncé à $65 \mathrm{~cm}$ de profondeur. Les différentes valeurs obtenues correspondent donc à la porosité locale entre 25 et $65 \mathrm{~cm}$ de profondeur (fig. $3 \mathrm{~d}$ ).

La porosité est plus élevée en amont des bancs de graviers (secteurs J, H, A) où elle est de l'ordre de
$30 \%$, qu'en aval (secteurs B et F) oì elle n'atteint que $24 \%$. Dans le chenal, la porosité décroît de l'amont (26\%) vers l'aval (21\%) : elle est toujours comparativement plus faible que dans le banc de rive gauche.

\subsection{La granulométrie}

Les données granulométriques ont été obtenues à partir des neuf échantillons prélevés pour l'étude de la porositél.

Les lutites $(<62,5 \mu \mathrm{m}$ ) représentent 1,2 à $4,9 \%$ des échantillons, les arénites $(62,5 \mu \mathrm{m}$ à $2 \mathrm{~mm}) 3$ à $11,9 \%$ et les rudites ( $>2 \mathrm{~mm}$ ) 84,3 à $93,4 \%$. Dans cette fraction grossière, les cailloux et les blocs ( $>$ $16 \mathrm{~mm}$ ) sont abondants puisque leur fréquence varie de 36,4 a $78,5 \%$. Le stock sédimentaire est donc diversifié avec une taille des grains qui s'étend des éléments fins à des blocs décimétriques.

Les caractéristiques des échantilions sont résumées dans le tableau I. Les médianes sont êlevées puisqu'elles sont d'ordre centimétrique. Les coefficients de Trask (So), net tement supérieurs à 1, indiquent un mauvais classement des sédiments. La concavité de type parabolique des courbes cumulatives semi-logarithmiques ( $f$ ig, 4), typique des alluvions des cours d'eau, confirme la nature peu évoluée des sédiments.

Les caractéristiques globales des échantillons indiquent clairement les affinités de certains d'entre eux ou, au contraire, leurs différences. Afin de mieux synthétiser la granulométrie du site étudié, une analyse factorielle des correspondances, complétée par une classification automatique (par agrégation autour de centres mobiles), a été réaljsée sur un tableau de données composé par les

1. L'analyse de la granulométrie a été réalisẻe au Laboratoire de Géologie - Pétrologie de l'Université Paul-Sabatier a Toulouse par C. Lucas que je remercie bien vivement. 


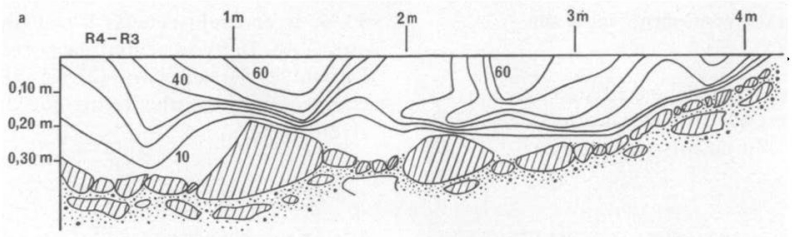

b
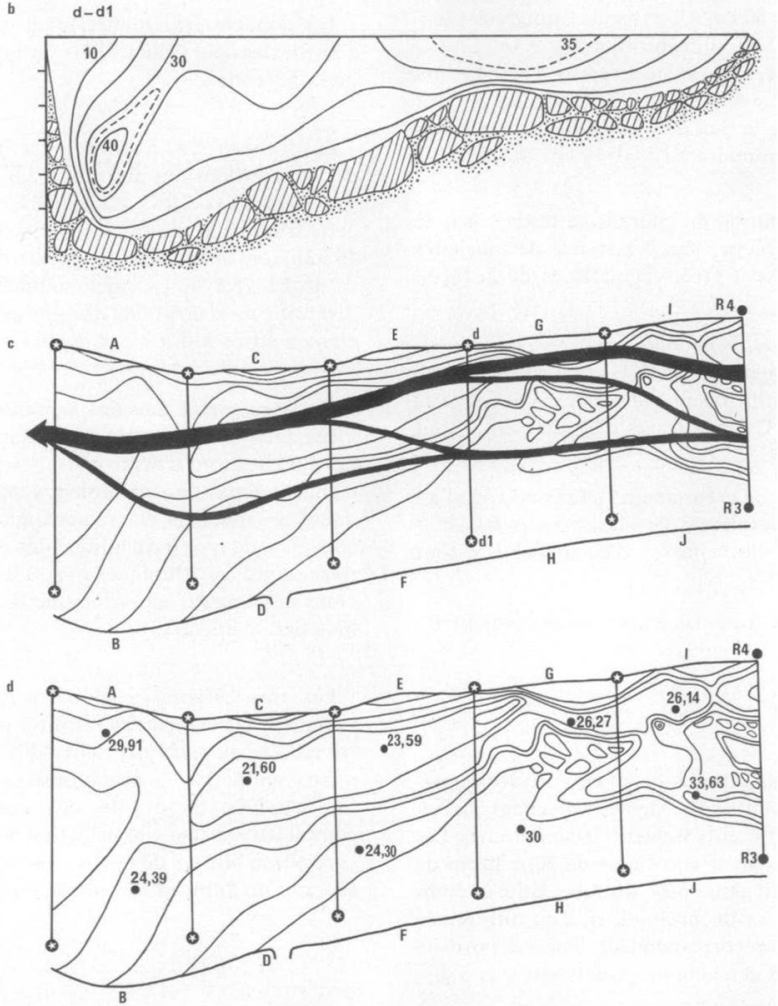

Fig. 3. Ruisseau de Luchein : a, b; profil des vitesses selon les lignes transversales $\mathbf{R} 4$ - R3 et d + d1 pour un débit de 0,345 ms ; $c$ : matérialisation des ecoulements principaux; $d$ : valeurs de la porosité (\%) en 9 points du site. 
Tableau I. Caractéristiques granulométriques des échantillons provenant des différents secteurs du tuisseau de Lachein.

\begin{tabular}{|c|c|c|c|c|}
\hline Echantilion & $\begin{array}{c}\text { Médiane } \\
\text { mm }\end{array}$ & $\begin{array}{c}\text { Q25 } \\
\text { un }\end{array}$ & So $=\left(\frac{Q 75}{Q 25}\right)^{0,5}$ \\
\hline A & 10 & 3.4 & 31 & 3.02 \\
\hline J & 8 & 3.4 & 34 & 3.16 \\
\hline H & 9.2 & 3.7 & 27.5 & 2.73 \\
\hline F & 27 & 4.9 & 69 & 3.75 \\
\hline B & 29 & 5.0 & 72 & 3.79 \\
\hline C & 22.5 & 9.2 & 55 & 2.44 \\
\hline G & 26 & 6.2 & 47 & 2.75 \\
\hline I & 25 & 7.0 & 45 & 2.53 \\
\hline E & 57 & 21.5 & 90 & 2.04 \\
\hline
\end{tabular}

9 échantillons et 10 variables (masse des particules $\mathrm{X} 1<62,5 \mu \mathrm{m}, 62,5<\mathrm{X} 2<125 \mu \mathrm{m}, 125<\mathrm{X} 3<$ $250 \mu \mathrm{m}, 250<\mathrm{X} 4<500 \mu \mathrm{m}, 0,5<\mathrm{X} 5<1 \mathrm{~mm}, 1$ $<\mathrm{X} 6<2 \mathrm{~mm}, 2<\mathrm{X} 7<4 \mathrm{~mm}, 4<\mathrm{X} 8<8 \mathrm{~mm}$, $8<\mathrm{X} 9<16 \mathrm{~mm}, \mathrm{X} 10>16 \mathrm{~mm}$ ).
La structure mise en évidence est forte (fig. 5) puisque les deux premiers facteurs rendent compte de $90,4 \%$ de la variance totale.

L'axe I permet de séparer les échantillons de I'amont des bancs de graviers (A, J, H), riches en éléments de 1 à $8 \mathrm{~mm}(\mathrm{X} 6, \mathbf{X} 7, \mathbf{X 8})$, d'un échantillon provenant du chenal (E), où les éléments supérieurs à $16 \mathrm{~mm}$ (X10) sont très abondants. L'axe II oppose un échantillon du chenal (C), où les éléments de 8 à $16 \mathrm{~mm}(X 9)$ sont importants aux échantillons de l'aval du banc de graviers de la rive gauche (B, F) où ces éléments sont mal représentés.

Trois groupements peuvent être reconnus. Le groupement $A, J, H$ rassemble les échantillons issus de l'amont des bancs de graviers : ils sont caractérisés par leur pourcentage élevé en sables grossiers, granules et gravillons et par leur faible pourcentage en graviers et cailloux. Le groupement C, E, G, I correspond aux échantillons du chenal riches en graviers et cailloux. Le groupement $B$, F correspond aux échantillons provenant de l'aval des bancs de

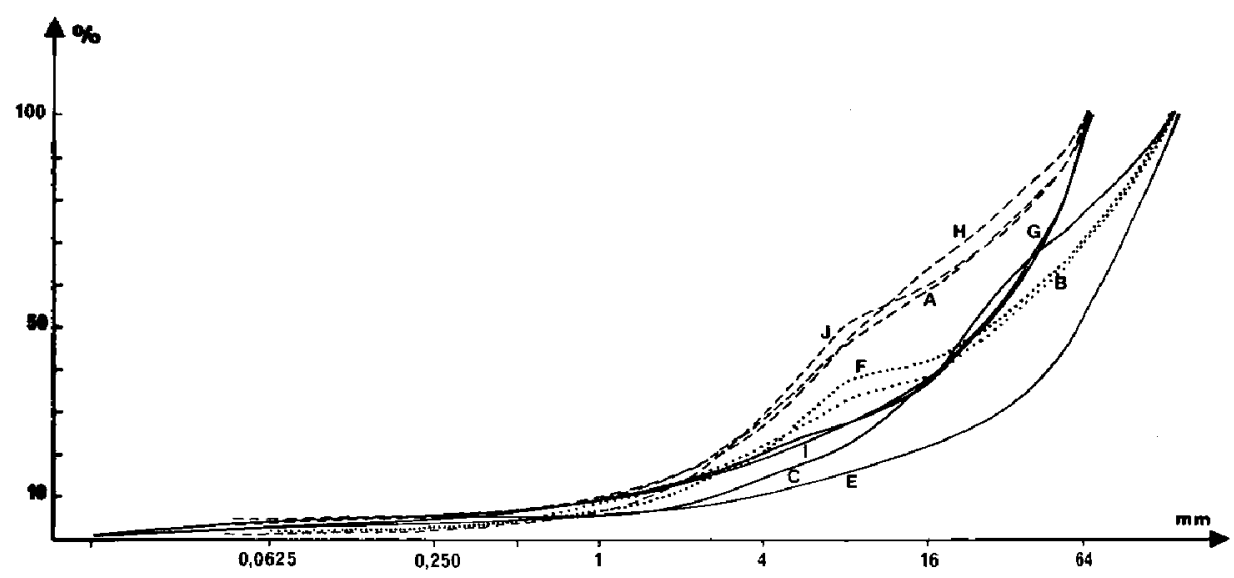

Fig. 4. Courbes granulométriques établies à partir des échantillons prélevés pour l'étude de la porosité. Chaque échantillon est identique par la lettre du secteur dont il est issu. 


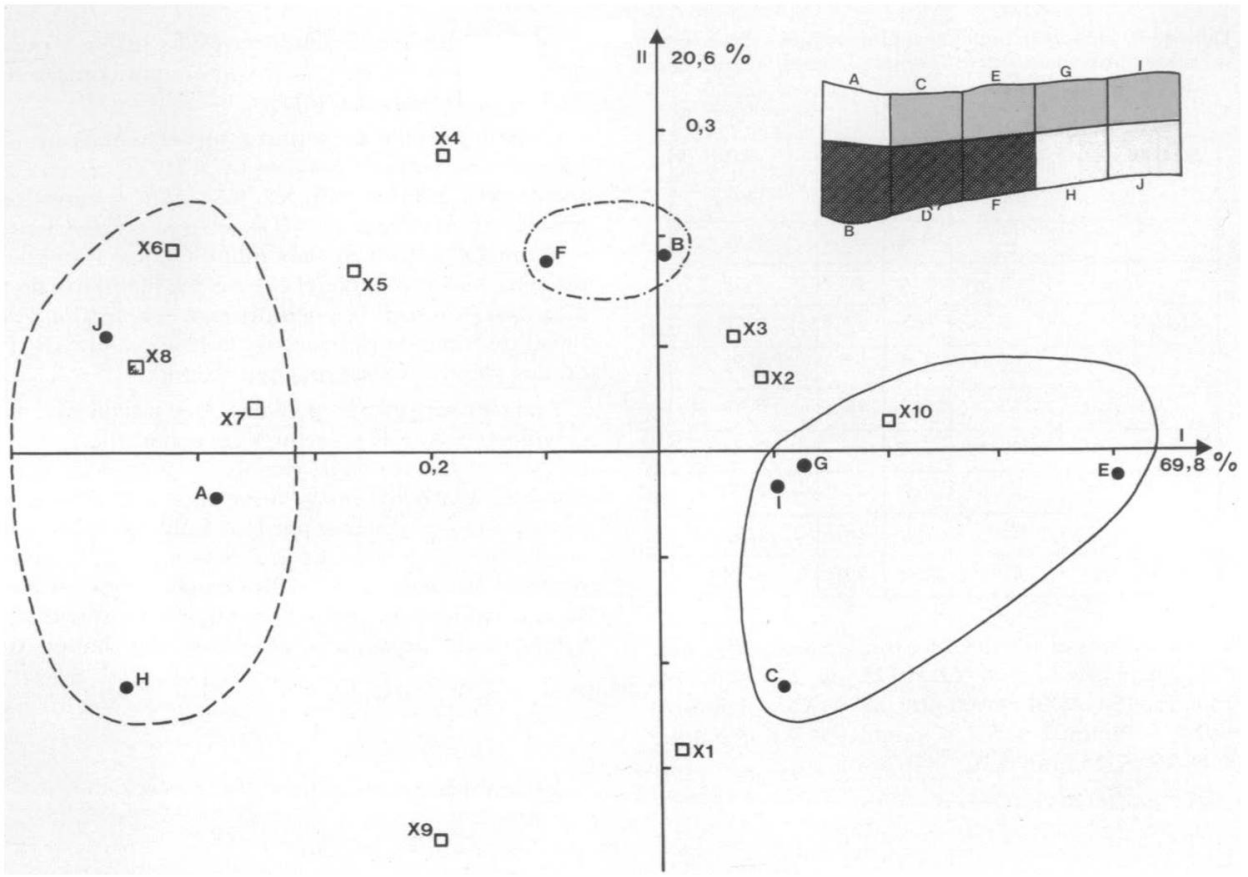

Fig. 5. Analyse factorielle des correspondances sur les neufs echantillons granulométriques. Points noirs : échantillons identifiés par la lettre du secteur dont ils sont issus. Carrés blancs : masses des particules selon leur taille (voir texte). Les trois ensembles mis en évidence ont été reportés sur la carte du site.

graviers : plus riches en sables grossiers et gravillons que le chenal et en graviers et cailloux que l'amont des bancs, ils occupent une place intermé. diaire entre les deux groupements précédents.

Ainsi, la distribution de la granulométrie reflète très directement celle des écoulements de surface puisque les différentes zones définies par ceux-ci présentent des profils distincts. Dans la relation porosité-granulométrie, on notera que ce sont les secteurs les plus riches en sables grossiers et gravillons (1 à $8 \mathrm{~mm}$ ) à l'amont des bancs, qui ont la meilleure porosité, ceux où prédominent les graviers et les cailloux, le chenal et l'aval des bancs, étant nettement moins poreux.

\subsection{La température}

De 1947 à 1985, la température moyenne de la source du ruisseau de Lachein (Andrieux, in litt.) a été de $10,09^{\circ} \mathrm{C}(\sigma=0,062 \cdot$ coefficient de variation $0.6 \%$ ). Pendant cette période, le minimum enregistré a été de $8,95^{\circ} \mathrm{C}$, le maximum de $10,84^{\circ} \mathrm{C}$, soit une amplitude de $1,89^{\circ} \mathrm{C}$ seulement. Cette grande homogénéité des températures à la source (elle assure l'essentiel des débits du cours d'eau) est à l'origine des variations relativement faibles observées sur le ruisseau, $800 \mathrm{~m}$ en aval (Tableau II et fig. 6). Les températures moyennes du ruisseau sont comprises entre $9,10^{\circ} \mathrm{C}$ et $11,3^{\circ} \mathrm{C}$ en surface, entre $9,0^{\circ} \mathrm{C}$ et $12,3^{\circ} \mathrm{C}$ dans le sous-écoulement. Au cours de l'année, les températures des deux milieux évo- 
Tableau II. Température moyenne $(\bar{\theta})$ du rüsseau de Lachein (R) et de son sous-écoulement (S); $\mathbf{n}=$ nombre de mestures ; $\sigma=$ écart type ; a $=$ amplitude.

\begin{tabular}{|c|c|c|c|c|c|c|c|c|}
\hline \multicolumn{2}{|c|}{ dates } & Sept 85 & Nov 85 & Fev 86 & Mars 86 & Mai 86 & Juil 86 & Sept 86 \\
\hline \multirow{3}{*}{$R$} & $n$ & 2 & 8 & 20 & 20 & 20 & 20 & 10 \\
\cline { 2 - 9 } & $\bar{\theta}$ & 11.05 & 9.10 & 9.50 & 9.80 & 11.00 & 11.00 & 11.30 \\
\cline { 2 - 9 } & $\sigma$ & 0.20 & 0.30 & 0.15 & 0.20 & 0.60 & 0.20 & 0.60 \\
\hline \multirow{5}{*}{$s$} & $n$ & 20 & 20 & 20 & 20 & 20 & 20 & 10 \\
\cline { 2 - 9 }$y$ & $\bar{\theta}$ & 12.30 & 9.00 & 9.25 & 9.90 & 11.30 & 11.60 & 11.40 \\
\cline { 2 - 9 }$y$ & $\sigma$ & 0.40 & 0.30 & 0.25 & 0.30 & 0.50 & 0.15 & 0.25 \\
\cline { 2 - 9 } & $\mathrm{a}$ & 1.30 & 1.30 & 0.90 & 0.65 & 1.80 & 0.75 & 0.90 \\
\hline
\end{tabular}

luent de façon parallèle, sans écart important. De mars à septembre elles sont plus élevées dans le sous-écoulement qu'en surface, et inversement en novembre et février.

Lors de chaque période d'échantillonnage, les valeurs ponctuelles de la température du sousécoulement n'ont jamais été uniformes, l'amplitude maximale des écarts allant de $0,65^{\circ} \mathrm{C}$ (mars) à $1,8^{\circ} \mathrm{C}$ (maj).

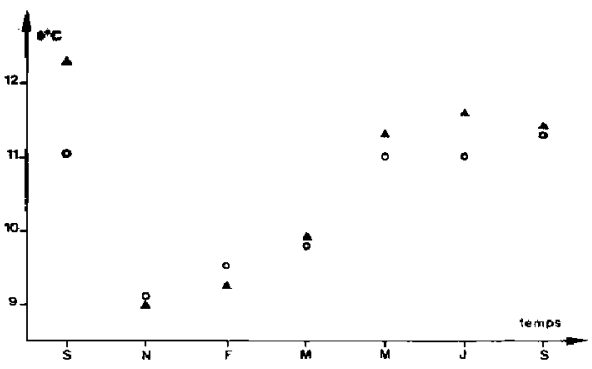

Fig. 6. Evolution de la température sur le ruisseau de Lachein: Triangles noirs: température moyenne du sous-écoulement. Cercles blancs: température moyenne des eaux de surface.

\section{Les caractéristiques chimiques}

4.1. Ions, conductivité, pH

Les données portent sur 137 prélèvements, 7 provenant du cours d'eau subaérien, 130 du sousécoulement. Afin d'établir les caractéristiques globales des eaux de surface comme celles de la profondeur, les différentes valeurs relatives au sousécoulement ont été exprimées sous forme de valeur moyenne pour l'ensemble des prélèvements effectués à une même date (Tableau III). L'analyse détaillée des valeurs ponctuelles fera l'objet d'une étude séparée.

\subsubsection{LE COURS D'EAU SUBAÉRIEN}

En raison de leur origine (cf. ante), les eaux de surface présentent des caractéristiques chimiques typiques d'un bassin versant calcaire. Il s'agit d'eaux bicarbonatées calciques où les bicarbonates représentent 70 à $89 \%$ du total des anions et le calcium plus de $80 \%$ du total des cations. L'ordre des cations $\mathrm{Ca}^{++}>\mathrm{Mg}^{++}>\mathrm{Na}^{+}>\mathrm{K}^{+}$et leur somme, $3100<\mathrm{SC}<3900 \mu \mathrm{eq} / \mathrm{l}$, de même que celui des anions, $\mathrm{HCO}_{3}^{-}>\mathrm{SO}_{4}^{-}>\mathrm{CI}^{-}$, sont caractéristiques des bassins versan ts calcaires ainsi 
Tableau III. Caractéristiques chimiques du ruisseau de Lachein (R) et de son sous-écoulement (S); $\mathrm{x}=$ valeur moyenne : $\sigma=$ écart-type.

\begin{tabular}{|c|c|c|c|c|c|c|c|c|c|c|c|c|c|}
\hline dates & & & $\mathrm{pH}$ & $\begin{array}{c}\mathrm{Ca} \\
\mathrm{mg} / 1\end{array}$ & $\underset{\mathrm{mg} / 1}{\mathrm{Mg}}$ & $\begin{array}{c}\mathrm{Na} \\
\mathrm{me} / \mathrm{l}\end{array}$ & $\mathrm{K}$ & $\begin{array}{l}\mathrm{SiO} 2 \\
\mathrm{mg} / \mathrm{l}\end{array}$ & $\begin{array}{r}\mathrm{NO}_{3} \\
\mathrm{mg} / 1\end{array}$ & $\begin{array}{c}\mathrm{Cl} \\
\mathrm{mg} / \mathrm{l}\end{array}$ & $\begin{array}{r}\mathrm{SO}_{4} \\
\mathrm{mg} / 1\end{array}$ & $\begin{array}{l}\mathrm{HCO}_{3} \\
\mathrm{mg} / 1\end{array}$ & $\begin{array}{l}\text { Cond } \\
\mu \mathrm{S} / \mathrm{cm}\end{array}$ \\
\hline Sept 85 & $\begin{array}{l}R \\
S\end{array}$ & $\begin{array}{l}\mathbf{x} \\
\boldsymbol{\sigma}\end{array}$ & $\begin{array}{l}8.30 \\
7.86 \\
0.15\end{array}$ & $\begin{array}{l}66.1 \\
66.7 \\
1.89\end{array}$ & $\begin{array}{l}6.60 \\
6.25 \\
0.31\end{array}$ & $\begin{array}{l}1.50 \\
1.45 \\
0.10\end{array}$ & $\begin{array}{l}0.70 \\
0.61 \\
0.07\end{array}$ & $\begin{array}{l}2.7 \\
2.2 \\
1.45\end{array}$ & $\begin{array}{l}1.19 \\
0.05 \\
0.07\end{array}$ & $\begin{array}{l}2.05 \\
1.75 \\
0.07\end{array}$ & $\begin{array}{l}53.5 \\
46.5 \\
2.47\end{array}$ & $\begin{array}{r}164.7 \\
171.6 \\
7.05\end{array}$ & $\begin{array}{r}315 \\
316 \\
4.31\end{array}$ \\
\hline Nov 85 & $S$ & $\begin{array}{l}x \\
\sigma\end{array}$ & $\begin{array}{l}8.16 \\
7.81 \\
0.14\end{array}$ & $\begin{array}{l}63.5 \\
63.9 \\
1.13\end{array}$ & $\begin{array}{l}6.20 \\
6.15 \\
0.27\end{array}$ & $\begin{array}{l}1.25 \\
1.20 \\
0.04\end{array}$ & $\begin{array}{l}0.56 \\
0.70 \\
0.10\end{array}$ & $\begin{array}{l}2.4 \\
2.5 \\
1.08\end{array}$ & $\begin{array}{l}1.94 \\
0.69 \\
0.62\end{array}$ & $\begin{array}{l}2.20 \\
2.25 \\
0.11\end{array}$ & $\begin{array}{l}37.0 \\
36.0 \\
2.19\end{array}$ & $\begin{array}{r}170.8 \\
176.9 \\
6.34\end{array}$ & $\begin{array}{r}289 \\
293 \\
3.58\end{array}$ \\
\hline Eev & $\mathrm{S}$ & $\begin{array}{l}x \\
\sigma\end{array}$ & $\begin{array}{l}8.21 \\
7.69 \\
0.07\end{array}$ & $\begin{array}{l}60.8 \\
62.4 \\
1.26\end{array}$ & $\begin{array}{l}4.70 \\
4.50 \\
0.39\end{array}$ & $\begin{array}{l}1.20 \\
1.40 \\
0.58\end{array}$ & $\begin{array}{l}0.74 \\
0.46 \\
0.12\end{array}$ & $\begin{array}{l}1.7 \\
2.2 \\
1.10\end{array}$ & $\begin{array}{l}0.03 \\
0.17 \\
0.24\end{array}$ & $\begin{array}{l}2.10 \\
2.10 \\
0.07\end{array}$ & $\begin{array}{l}21.0 \\
20.0 \\
2.17\end{array}$ & $\begin{array}{r}176.3 \\
183.5 \\
3.66\end{array}$ & $\begin{array}{r}279 \\
285 \\
4.49\end{array}$ \\
\hline Mars 86 & $\begin{array}{l}R \\
S\end{array}$ & $\begin{array}{l}x \\
\sigma\end{array}$ & $\begin{array}{l}8.10 \\
7.70 \\
0.15\end{array}$ & $\begin{array}{l}54.9 \\
56.8 \\
2.03\end{array}$ & $\begin{array}{l}4.60 \\
4.45 \\
0.14\end{array}$ & $\begin{array}{l}1.10 \\
1.30 \\
0.15\end{array}$ & $\begin{array}{l}0.53 \\
0.47 \\
0.11\end{array}$ & $\begin{array}{l}2.6 \\
3.3 \\
0.45\end{array}$ & $\left|\begin{array}{l}0.78 \\
2.08 \\
0.81\end{array}\right|$ & $\begin{array}{l}1.55 \\
1.70 \\
0.07\end{array}$ & $\begin{array}{l}13.7 \\
14.0 \\
1.38\end{array}$ & $\begin{array}{r}162.3 \\
168.7 \\
8.47\end{array}$ & $\begin{array}{r}254 \\
261 \\
9.65\end{array}$ \\
\hline Mai 86 & $\begin{array}{l}\mathrm{R} \\
\mathrm{S}\end{array}$ & $\begin{array}{l}\mathbf{x} \\
\sigma\end{array}$ & $\begin{array}{l}8.22 \\
7.83 \\
0.11\end{array}$ & $\begin{array}{l}57.6 \\
58.7 \\
1.85\end{array}$ & $\begin{array}{l}4.20 \\
4.65 \\
0.91\end{array}$ & $\begin{array}{l}1.30 \\
1.40 \\
0.02\end{array}$ & $\begin{array}{l}0.40 \\
0.51 \\
0.09\end{array}$ & $\mid \begin{array}{l}3.2 \\
3.3 \\
0.42\end{array}$ & $\begin{array}{l}1.00 \\
1.15 \\
0.33\end{array}$ & $\begin{array}{l}1.50 \\
1.50 \\
0.11\end{array}$ & $\begin{array}{l}17.0 \\
14.0 \\
1.44\end{array}$ & $\begin{array}{r}161.0 \\
169.2 \\
6.32\end{array}$ & $\begin{array}{r}263 \\
272 \\
5.89\end{array}$ \\
\hline Juil 86 & $\begin{array}{l}\mathbf{R} \\
\mathbf{S}\end{array}$ & $\begin{array}{l}x \\
\sigma\end{array}$ & $\begin{array}{l}8.15 \\
7.77 \\
0.15\end{array}$ & $\begin{array}{l}57.7 \\
59.0 \\
1.52\end{array}$ & $\begin{array}{l}4.90 \\
4.80 \\
0.17\end{array}$ & $\begin{array}{l}1.02 \\
1.00 \\
0.02\end{array}$ & $\begin{array}{l}0.42 \\
0.51 \\
0.10\end{array}$ & $\begin{array}{l}3.0 \\
3.5 \\
0.27\end{array}$ & $\begin{array}{l}1.95 \\
2.07 \\
0.61\end{array}$ & $\begin{array}{l}1.50 \\
1.45 \\
0.07\end{array}$ & $\begin{array}{l}24.0 \\
23.5 \\
2.37\end{array}$ & $\begin{array}{r}159.8 \\
163.1 \\
6.55\end{array}$ & $\begin{array}{r}266 \\
272 \\
5.98\end{array}$ \\
\hline Sept 86 & $\begin{array}{l}\mathrm{R} \\
\mathrm{S}\end{array}$ & $\begin{array}{l}\mathbf{x} \\
\sigma\end{array}$ & $\begin{array}{l}8.20 \\
7.78 \\
0.12\end{array}$ & $\begin{array}{l}62.1 \\
63.7 \\
1.60\end{array}$ & $\begin{array}{l}5.60 \\
5.60 \\
0.16\end{array}$ & $\begin{array}{l}1.12 \\
1.10 \\
0.04\end{array}$ & $\begin{array}{l}0.62 \\
0.60 \\
0.06\end{array}$ & $\begin{array}{l}3.6 \\
4.0 \\
0.28\end{array}$ & $\begin{array}{l}4.80 \\
2.63 \\
0.52\end{array}$ & $\begin{array}{l}1.70 \\
1.95 \\
0.36\end{array}$ & $\begin{array}{l}37.0 \\
37.0 \\
0.93\end{array}$ & $\begin{array}{r}157.4 \\
162.7 \\
4.92\end{array}$ & $\begin{array}{r}289 \\
297 \\
5.66\end{array}$ \\
\hline
\end{tabular}

que la conductivité comprise entre 250 et $315 \mu \mathrm{S} / \mathrm{cm}$.

Les teneurs en sulfate ( 13,7 à $53,5 \mathrm{mg} / \mathrm{l})$ soit 9,5 à $28,7 \%$ du total des anions sont plus élevées que la normale, inférieure à $8 \%$ selon Meybeck (1984). Elles sont également supérieures à celles de l'aquifère du Baget (Bakalowicz 1979). Ce phénomène est dû aux apports du petit affluent de rive gauche, le ruisseau de Plamicou, qui draine des argiles et des marnes gypsifères du Trias.

\subsubsection{LE SOUS-ÉCOULEMENT}

Ses caractéristiques chimiques sont voisines de celles du flux subaérien ce qui traduit l'importance des échanges entre la surface et la profondeur. Ainsi les teneurs en $\mathrm{Ca}^{+}+, \mathrm{Mg}^{++}, \mathrm{SiO}_{2}, \mathrm{Cl}-$ et $\mathrm{SO}_{4}-\mathrm{du}$ sous-écoulement de méme que la conductivité diffèrent peu de celles de la surface quelle que soit l'époque de l'année. Seuls le $\mathrm{pH}$ et les bicarbonates montrent des différences constantes. Le pH est toujours moins élevé en profondeur qu'en surface avec des valeurs moyennes comprises entre 7,69 et 7,86. Inversement, les teneurs en bicarbonates sont toujours plus fortes de 5 a $7 \mathrm{mg} / \mathrm{l}$ dans le sousécoulement.

En profondeur, anions et cations suivent une évolution annuelle identique à celle observée pour les eaux de surface. 


\subsection{L'oxygène dissous}

Les mesures d'oxygène dissous ne concernent que cinq périodes d'échantillonnage : novembre 1985 , mars, mai, juillet, septembre 1986.

81 mesures ont été réalisées dans les eaux de surface soit, respectivement, 17, 14, 20, 20 et 10 mesures aux périodes précitées, avec pour valeur moyenne $96 \%(\sigma=4,72)$, sans qu'aucun cycle saisonnier apparaisse. Dans le méme temps, 83 mesures étaient effectuées dans le sous-écoulement ( 19 , $14,20,20,10)$. Les valeurs y sont toujours inférieures à celles observées en surface; en outre, elles sont extrèmement hétérogènes $(\bar{x}=62.5 \%, \sigma=21,06)$. Toutefois, les cinq séries de mesures ne sont pas significativement différentes (test de Fisher: $\mathrm{F}=$ 0,11 pour $F 0,05=2,53$ avec 4 et 60 ddl) ce qui implique I'absence de cycle saisonnier. De plus, prises deux à deux, ces cinq séries de mesures ne montrent aucune différence significative.

Afin d'identifier les tendances générales de la distribution de l'oxygène dissous, les différentes valeurs de celui-ci ont fait l'objet d'une classifica- tion automatique a trois classes (agrégation autour de centres mobiles) : classe $\mathrm{I}, \mathrm{n}=12, \mathrm{O}_{2}$ moyen $=$ $16,2 \%, \sigma=12,7, \operatorname{mini}=0,02 \%, \operatorname{maxi}=36,2 \%$; classe $\Pi, n=51, O_{2}$ moyen $=74,3 \%, \sigma=4,46$, $\operatorname{mini}=67,4 \%$, $\operatorname{maxi}=84,7 \%$; classe III, $\mathrm{n}=20$, $\mathrm{O}_{2}$ moyen $=60 \%, \sigma=5,84, \mathrm{mini}=47,8 \%$, maxi $=67,1 \%$.

Les limites de ces classes, reportées sur la carte du site (fig. 7), montrent les faits suivants. Tous les prélèvements à faible teneur (classe I) proviennent de la zone d'eau morte définie par les écoulements (secteurs $\mathrm{B}$ et $\mathrm{D}$ ) : elle sera dérommée zone réductrice. Les prélèvements à teneurs élevées (classe II) sont issus du banc de graviers de la rive gauche (secteurs $\mathrm{J}, \mathrm{H}$ et une partie de $F, G, I$ ) et de celui de la rive droite (secteur A et une partie de $\mathrm{C}$ ). Les prélèvements a teneurs moyennes (classe III) proviennent de la zone où le chenal affouille la rive droite et de la périphérie des bancs de graviers.

Ainsi la distribution de l'oxygène dissous est liée à la dynamique des écoulements de surface puisqu'elle suit les limites physiographiques définies par ceux-ci.

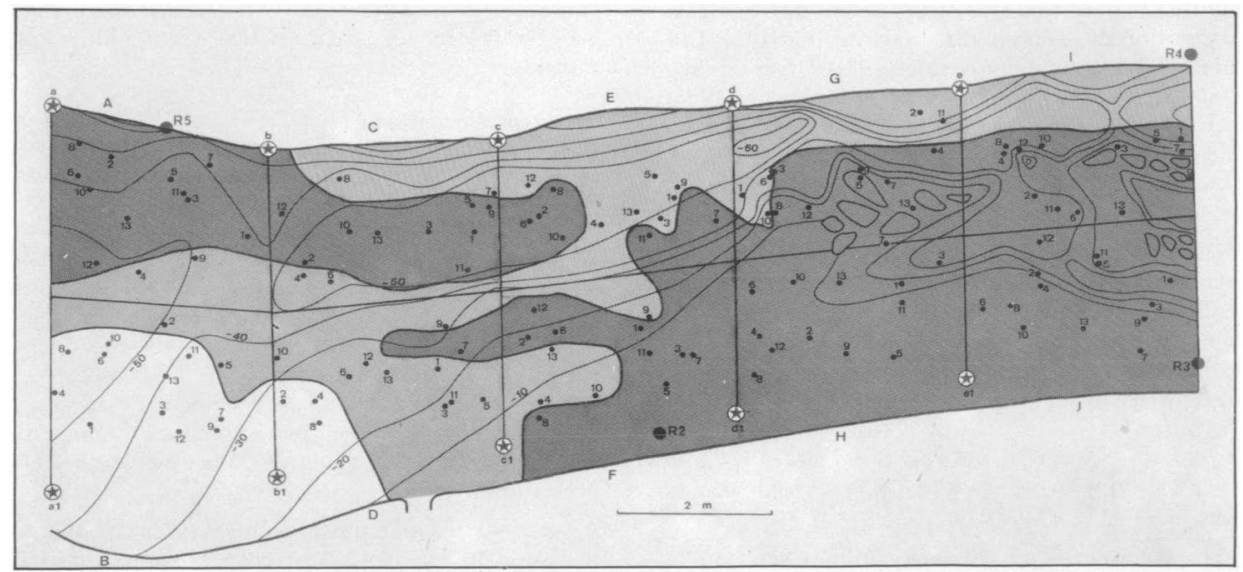

Fig. 7. Distribution de $\mathrm{l}^{\prime} \mathrm{O}_{2}$ dissous. Zone gris foncé : teneurs supérieures à $67 \%$. Zone gris clair : teneurs comprises entre 48 et $67 \%$. Zone claire : teneurs inférieures a $36 \%$. 


\subsection{La matière organique particulaire}

Elle a été estimée par la perte au feu des sédiments présents dans les 2,5 litres pompés à cet effet lors de chaque prélèvement. Après évaporation de l'échantillon prélevé, à $75^{\circ} \mathrm{C}$ pendant 48 heures, $1 \mathrm{~g}$ du résidu sec est porté à $540^{\circ} \mathrm{C}$ pendant deux heures : la quantité de matière organique correspond à la perte de poids de l'échantillon exprimée en pourcentage. Seuls les échantillons de septembre et novembre 1985, février, mars et mai 1986 ont été pris en compte, soit 100 au total. 87 d'entre eux ont fait l'objet d'une double mesure afin d'estimer la précision des résultats : le coefficient de variation de la moyenne de 68 d'entre eux est inférieur à 10 $\%$, celui de 15 est compris entre 10 et $20 \%$, celui de 4 est compris entre 20 et $30 \%$. Une part de la variabilité observée est donc liée aux conditions dans lesquelles la technique a été utilisée.

Les 187 dosages donnent une teneur moyenne de $8,17 \%(\sigma=1,641 ; \mathrm{cv}=20 \%)$, avec pour valeurs extrêmes $4,44 \%$ (B6) et 13,60\% (A2). Cet te for te hétérogénéité des teneurs a une double origine, spatiale et temporelle. En effet, les cinq séries de mesures sont très significativement différentes (test de Fisher : $F=22,25$ avec $F 0,01=3,32$ pour 4 et $182 \mathrm{dl}$ ). Prises deux à deux, ces séries sont également très significativement ou significativement différentes à l'exception de la comparaison mars - mai 1986. Les valeurs des teneurs moyennes: $9,8 \%$ (septembre 1985) - 8,7\% (novembre 1985) - 6,9\% (février 1986) $-8,1 \%$ (mars 1986) $-7,7 \%$ (mai 1986) indiquent donc une évolution saisonnière où les teneurs les plus faibles coïncident avec la période de hautes eaux.

Du point de vue spatial, les 10 séries de prélèvements relatives aux différents secteurs de la zone étudiée sont très significativement différentes (test de Fisher : $F=4,00$ avec $F 0,01=2,41$ pour 9 et 177 ddl). En valeur moyenne, la distribution de la matière organique peut être schématisée de la façon suivante :

$\mathbf{A}=8,7 \% ; \mathbf{C}: 9,4 \% ; \mathbf{E}=7,3 \% ; \mathbf{G}=7,2 \%:[=7,8 \%$ $\mathbf{B}=8,3 \% ; \mathrm{D}=8,9 \% ; \mathrm{F}=8, \boldsymbol{0} \% ; \mathbf{H}=8.5 \% ; \mathbf{J}=7,7 \%$

Les valeurs les plus basses caractériseraient le chenal (E,G) et l'amont du site étudié ; les valeurs les plus élevées apparaissent dans les bancs de graviers.

\section{Le peuplement des Crustacés}

\subsection{Aspect faunistique}

Les Crustacés des eaux souterraines du système karstique du Baget et du ruisseau de Lachein sont au nombre de 63, dont 31 taxons stygobies. Ils appartiennent aux groupes suivants : Ostracodes, Copépodes (Cyclopides et Harpacticides), Syncarides, Isopodes et Amphipodes (tableau IV).

Chacun de ces deux milieux, karstique et interstitiel, est peuplé par 50 espèces et sous-especes de Crustacés avec, respectivement, 21 et 22 taxons stygobies. Toutes espèces confondues, le coefficient de similarité de Sorensen, $\mathrm{Sr}$, a pour valeur 0,740 ce qui traduit une grande similitude faunistique entre ces deux milieux. Celle-ci est due davantage à la forte proportion d'espèces épigées communes aux deux biotopes ( $\mathrm{Sr}=0,877$ ), qu'à celle des hypogées ( $\mathrm{Sr}=0,558$ ). C'est ainsi que les Ostracodes et les Cyclopides n'ont aucune espèce hypogée commune aux deux habitats. La similarité des peuplements chez ces deux groupes est donc liée aux espèces épigées. Avec les Harpacticides, la différenciation est moins net te : si la similarité faunistique est élevée pour les espèces hypogées $(\mathrm{Sr}=0,857)$, elle l'est encore davantage pour les espèces épigées ( $\mathrm{Sr}$ $=0,960$ ). 6 des 8 Malacostracés hypogés sont communs aux deux milieux, seul Stenasellus virei ayant des sous-espèces différentes selon l'habitat considéré.

\subsection{Aspect quantitatif}

Les Ostracodes n'ayant plus été isolés après mars 1986, seules les quatre premières séries de prélèvements ont été analysées globalement (tableau V). Les Copépodes, avec 94, $1 \%$ des captures, sont les Crustacés de loin les plus abondants et, notamment, les Harpacticides qui représentent $61,8 \%$ du peuplement carcinologique. Les Ostracodes viennent ensuite puis les divers groupes de Malacostracés. Les valeurs des pourcentages de chacun de ces groupes de Crustacés varient peu au cours du temps, si bien que la structure générale du peuplement paraît très stable.

Les densités globales moyennes pour ces quatre séries de prélèvements sont, respectivement, de 430 - 296 - 366 - 385 individus $/ 10$ litres. Les coefficients de variations élevés $(66,3 \%, 59 \%, 63,1 \%, 72,9 \%)$ affectant ces moyennes, traduisent la forte 
Tableau IV. Liste des Crustacés peuplant le sous-écoulement du ruisseau de Lachein et le système karstique du Baget : Ostracodes (dét. Danielopol), Cyclopides (dét. Lescher-Moutoué), Syncarides (dét. Serban), Isopodes (dét. Magniez et Coịneau), Amphipodes (dét. Bou).

\begin{tabular}{|c|c|c|}
\hline Espeoed & Lachain & Baget \\
\hline \multicolumn{3}{|l|}{ OSTRACODES } \\
\hline * Pseudiocandona sp 1 & & + \\
\hline * $\ldots \ldots \ldots \ldots$ sp. & & + \\
\hline$\star \ldots \ldots \ldots \ldots$ sp.3 & + & \\
\hline * .......... mouch & + & \\
\hline * $\ldots \ldots \ldots \ldots$ gp aff hergagi (Rlie, 1938) & + & \\
\hline ........ albicans (Brady, & + & + \\
\hline$\ldots \ldots \ldots \ldots$ ap aff. marchica (Kartwid, & + & + \\
\hline 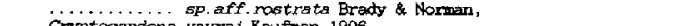 & + & \\
\hline Cryptocandans warral Raufmam, 1906 & + & + \\
\hline Candana neglects Sara, 1887 & + & + \\
\hline Cypria sp.aff. lacustris Sars, 1890 & + & + \\
\hline Cyclocypris sp. & + & + \\
\hline Iliocypris sp. & & + \\
\hline Elroppris pigra (Fisher, 1851) & + & \\
\hline Brychodromes fontinalis (Wolf, & + & + \\
\hline \multirow{3}{*}{\multicolumn{3}{|c|}{$\begin{array}{l}\text { GOPEPODES } \\
\text { Oyclopidee }\end{array}$}} \\
\hline & & \\
\hline & + & \\
\hline $\begin{array}{l}\text { bucylops serrulatus (Fleher, 1851\}) } \\
\text { Paracyclops finbrtatus (Figher, 1853) }\end{array}$ & + & + \\
\hline Tropocyclope prasinus (FtEher, 1860 ) & & + \\
\hline * Acanthocyelops deris 1tivis (Graeter \& Chappuis, 1914) & + & \\
\hline$\ldots \ldots \ldots \ldots \ldots$ viridis (Jurine,1820) & & + \\
\hline ........... venustus (Norman \& Scott, 1906) & + & + \\
\hline Diagralops bisetosus (Rehberg, 1880) & + & + \\
\hline ....... 1,anguidus beletcus Kiefer, 1936 & + & \\
\hline ........ langufdoides Lilljeborg, 1901 & + & + \\
\hline * Graeteriella rouch1 Lescher-Moutoue, 1968 & + & \\
\hline * ........ n, sp. & + & \\
\hline * Speocyclops racovitzai liqueraig Chapruis \& Riefer, 1952 & & + \\
\hline * ....... . . . . . . cf. boscoensis Klefer, 1954 & & + \\
\hline * . . . . . . ancma lus Chappate \& Rlefer, 1952 & & + \\
\hline * $\ldots \ldots \ldots \ldots$. kieferi Leecher-Moutoue, 1968 & & + \\
\hline \multicolumn{3}{|l|}{ Harpactletdes } \\
\hline Furzlogna thopus rieuteri Malupas, 1892 & + & + \\
\hline * Parapeudoleptomesochra subterranes (Chappule, 1928) & + & + \\
\hline * Nitocrella stacilia Ghappuis, 1955 & + & + \\
\hline * . . . . . . delayi Rouch, 1970 & & + \\
\hline At theyella crassa (Sars, 1863) & + & + \\
\hline Maraenobiotus Nejolovaky 1 Mrazek, 1893 & & + \\
\hline Fpactophanes richardi Mrazek, 1893 & + & + \\
\hline * Mararta catalana Chappule \& Kiefer.1952 & + & + \\
\hline ..... pectinata Thiebaud \& Pelosee, 1928 & + & + \\
\hline . . . . . poppel (Mrazek, 1893) & + & + \\
\hline$\ldots \ldots \ldots$ varfoa (Graeter, 1911) & + & + \\
\hline * Ceuthonectes gallicus Chappuib, 1928 & $*$ & + \\
\hline Bryocanptus zechokkel (Schme11, 1893) & 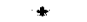 & + \\
\hline$\ldots . . . . .$. pyemraus (Sars, 1863) & + & + \\
\hline$\ldots \ldots \ldots$ minutus (Claus, 1863 ) & + & + \\
\hline$\ldots . . .$. typhlops (Mrazele, 1893) & + & + \\
\hline$\ldots \ldots \ldots$ (L.) ochinatus (Hrazok, 1893) & + & + \\
\hline * Blaphoidella bouilitant Rouch, 1964 & 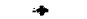 & $*$ \\
\hline * ........ coiffaiti Chappuis \& Kiefer, 1952 & 4 & + \\
\hline * Antrccamptus catherinae Chappuie \& Rouch, 1960 & & + \\
\hline * ... . . . . chapputst Rouch, 1970 & + & \\
\hline Paracamptus schanelli (Mrazei, 1893) & 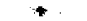 & + \\
\hline * Parastenocarls dianse Chappulo, 1955 & + & \\
\hline * ......... vasdell Rouch, 1988 & + & \\
\hline \multicolumn{3}{|l|}{ STMCARIDES } \\
\hline * Vandelihathymella vandelf (Delamare Deboutiteville \& Chappuis, 1854) & + & + \\
\hline * Stenagellus virel husson 1 Magniez, 1968 & & + \\
\hline 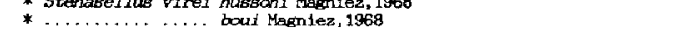 & + & + \\
\hline * Microcharar rocuhi Coineai, 1968 & 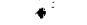 & + \\
\hline \multicolumn{3}{|l|}{ ANIFHIPODES } \\
\hline * Nlphargus kophianus Bate, 1859 & + & + \\
\hline * Salentinella petiti Coinead. 1968 & + & + \\
\hline * Parasalertinelia rouchl Bou, 1971 & + & + \\
\hline * Insolfiel le thibridt Colread, 1968 & + & + \\
\hline Stysobies & 22 & 21 \\
\hline Epigeos & $\mathbf{2 8}$ & 29 \\
\hline Total & 50 & 50 \\
\hline
\end{tabular}


Tableau V. Nombre total d'individus capturés (n) et leur pourcen 1985 a mars 1986).

irs de chaque série de prélèvements (Septembre

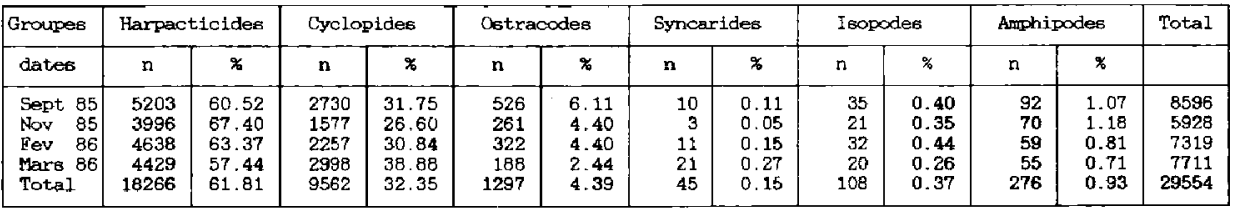

hétérogénéité des densités ponctuelles dont les valeurs extrêrnes sont de 10 et $1081 \mathrm{i} / 10 \mathrm{l}$. Toutefois, il n'existe aucune fluctuation saisonnière significative des densités entre ces quatre séries de 20 prélèvements (test de Kruskal - Wallis : $\mathrm{H}=2,35$ pour $\mathrm{X}^{2} 0,05=7,82$ avec $3 \mathrm{dd}$ ).

\subsection{Les Malacostracés}

Capturés lors de chaque période d'échantillonnage, les Syncarides, les Isopodes et les Amphipodes sont des hôtes permanents du sous-écoulement dans le site étudié (Tableau VI).

Seule Ingolfiella thibaudi est une espèce constante (fréquence $=77,7 \%$ ). Stenasellus virei bout est commun (fréquence $=\mathbf{4 4 , 6} \%$ ), alors que Vandelibathynella vandeli ( $\mathrm{f}=6,2 \%$ ) Microcharon rouchi (f $=20,8 \%)$ Niphargus kochianus $(\mathrm{f}=18,5 \%)$ Saleninella petiri (f $=22,3 \%$ ) et Parasalentinella rouchi (f $=16,9 \%$ ) sont rares.

Toutes ces espèces sont peu abondantes, avec des densités moyennes comprises entre 2 et 8 individus $/ 101$, compte tenu des stations où elles sont présentes.

Leur repartition n'est pas uniforme (fig. 8). Stenasellus, Niphargus et Ingolfiella vivent sur l'ensemble du site, à l'exclusion de la zone réductrice. Salentinella et parasalentinella occupent le sousécoulement du chenal et la périphérie des bancs de graviers. Microcharon colonise uniquement les bancs de graviers. Vandelibathynella est confinée dans la zone réductrice.

Tableau VI. Malacostracés : nombre total d'individus capturés (n) et nombre de stations (st) où les espèces étaient présentes lors de chaque série de prélèvements. Densité moyenne (d/10 l) et pourcentage moyen des stations occupées par les différentes especes (fréquence \%).

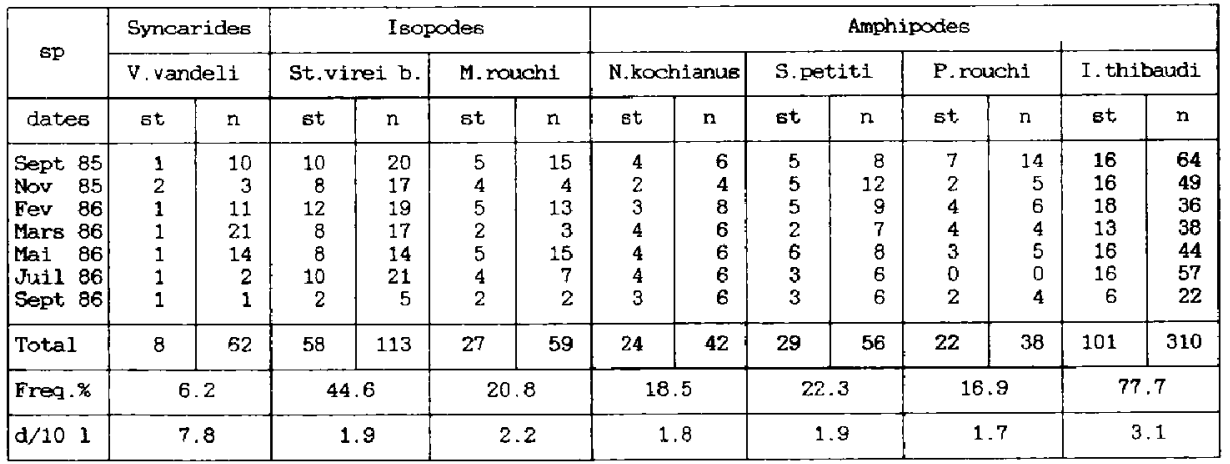



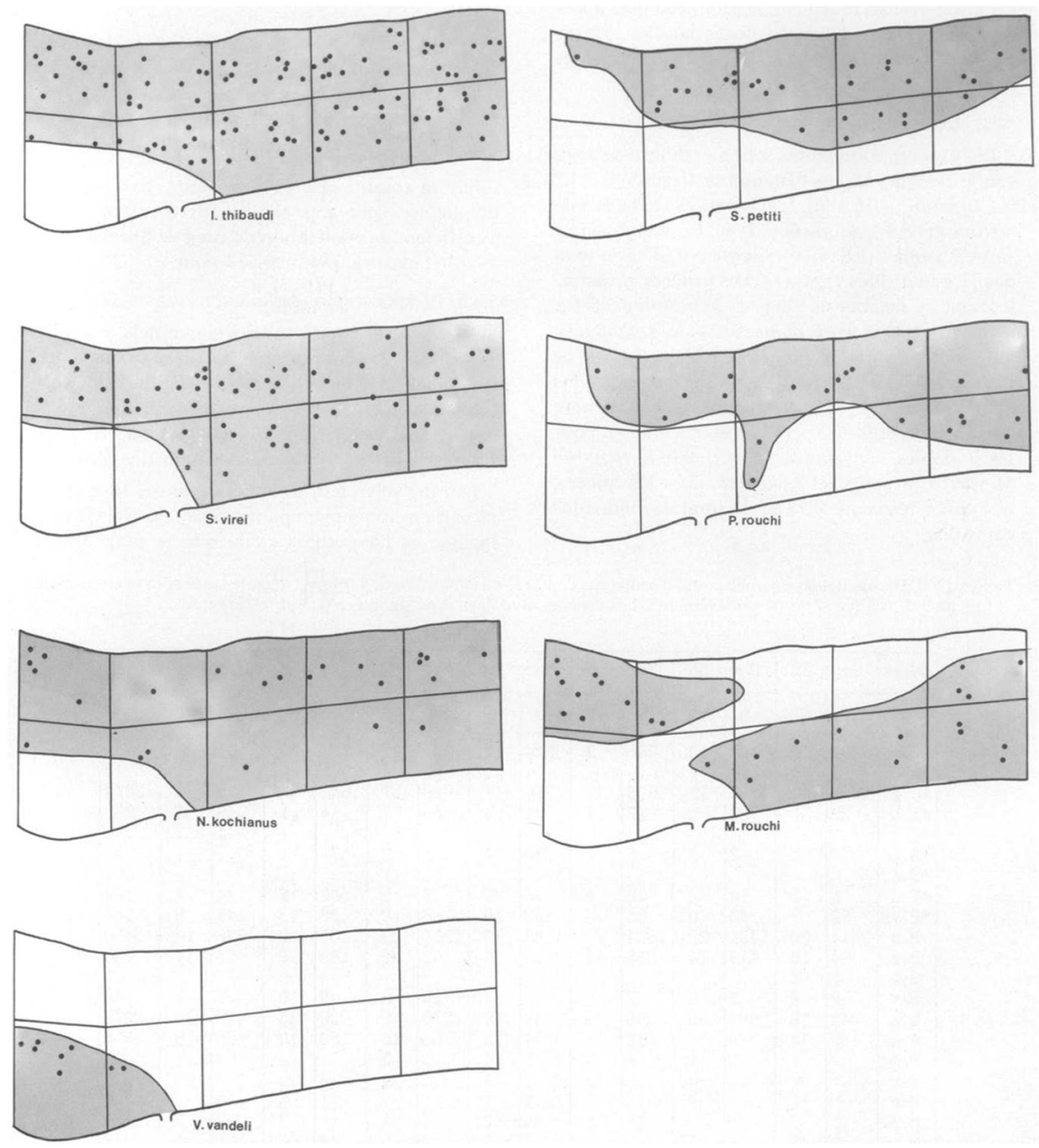

Fig. 8. Répartition des Majacostracés. Les zones colonisées sont en grisé ; chaque point correspond à un prétèvement positif. 


\section{5,4, Les Harpacticides}

Ils sont, numériquement, le groupe le plus abondant avec 29777 individus capturés dans les 130 prélèvements et appartiennent à 21 espèces, 9 stygobies et 12 épigées (Tableau VII).

\subsubsection{DOMINANCES ET FRÉOUENCES GLOBALES}

Les espèces dominantes sont au nombre de sept, soit le tiers des taxons dénombrés. C. gallicus $(43,2$ $\%)$, B. minutus $(35,1 \%)$, L. echinatus $(7,7 \%)$, P. subterranea $(4,8 \%)$, A. crassa $(3,1 \%)$, B. zschokkei $(2,1$ $\%$ ) et $P$. vandeli $(1,8 \%)$ représentent $97,8 \%$ du total des Harpacticides capturés. Les espèces principales sont au nombre de cinq: E. bouilloni $(0,88 \%)$, M. pectinata $(0,53 \%)$, P. dianae $(0,34 \%)$, A. chappuisi $(0,13 \%)$ et $B$. typhlops $(0,13 \%)$ forment $2,02 \%$ de l'ensemble des individus. Les espèces les moins abondantes $(0,003 \%<x<0,04 \%)$ sont au nombre de neuf : E. coiffaiti, $N$. gracilis et $M$. catalana pour les stygobies, B. pygmaeus, $P$. schmeili, E. richardi, $M$. varica, $M$. poppei et $P$. viguieri, pour les épigées, ne représentent que $0,13 \%$ du total des individus capturés.
Les espèces constantes correspondent aux sept espèces dominantes: $B$. minutus (fréquence $=99,2$ $\%)$, L. echinattrs $(94,6 \%), B$. zschokkei $(85,4 \%), C$. gallicus $(85,4 \%)$, A. crassa $(83,8 \%)$, P. subterranea $(52,3 \%)$ et $P$. vandeli $(51,5 \%)$. Toutes les autres espèces (14) sont rares (fréquence inférieure à $25 \%$ ). Tou. tefois les espèces principales sont plus fréquentes que les espèces les moins abondantes.

Sur la totalité des prélèvements, fréquence et dominance sont très significativement corrélées (coefficient de corrélation de rang de Spearman rs $=0,964$ avec $\mathrm{rs} 0,01=0,534$ pour $\mathrm{n}=20$ ).

\subsubsection{DOMINANCES ET FRÉQUENCES PARTIELLES}

A l'échelle des prélèvements bimensuels, seules 10 especes sur 21 sont présentes de façon permanente. Il s'agit des 7 espèces dominantes définies antérieurement ainsi que des 3 espèces principales $E$. bouilIoni, P. dianae et B. typhlops (voir Tableau VII). Elles représentent $99,2 \%$ du total des individus capturés.

Le rang selon leur dominance de ces 10 espèces ne varie pratiquement pas au cours du cycle hydrologique et l'hypothèse nulle que le rang de ces

Tableau VII. Harpacticides : nombre total d'individus capturés (n) et nombre de stations (st) oủ les espèces étaient présentes lors de chaque série de prélèvements. Les espèces stygobies sont signalees par un astérisque.

\begin{tabular}{|c|c|c|c|c|c|c|c|c|c|c|c|c|c|c|c|}
\hline \multirow{2}{*}{\begin{tabular}{|c|} 
dates \\
sp.
\end{tabular}} & \multicolumn{2}{|c|}{ Sept 85} & \multicolumn{2}{|l|}{ Nov } & \multicolumn{2}{|c|}{ Fev 86} & \multicolumn{2}{|c|}{ Mars 86} & \multicolumn{2}{|c|}{ Mai 86} & \multicolumn{2}{|c|}{ Juil 86} & \multicolumn{2}{|c|}{ Sept 86} & \multirow[t]{2}{*}{ Total } \\
\hline & $\mathrm{n}$ & st & $\mathrm{n}$ & st & n & st & $\mathrm{n}$ & st & $\mathbf{n}$ & st & $\mathbf{n}$ & st & $\mathrm{n}$ & st & \\
\hline *P. 6 & 299 & 10 & 100 & 10 & 295 & 9 & 224 & 11 & 123 & 11 & 297 & 11 & 101 & 6 & 1439 \\
\hline *N, $\mathrm{g}$ & & & & & & & & & 13 & 2 & & & & & 13 \\
\hline *C.g & 1913 & 17 & 1658 & 17 & 1988 & 17 & 2286 & 17 & 1620 & 17 & 1963 & 15 & 1446 & 9 & 12874 \\
\hline$* \mathrm{E} . \mathrm{b}$ & 22 & 1 & 90 & 2 & 12 & 1 & 31 & 4 & 58 & 2 & 4 & 1 & 46 & 1 & 263 \\
\hline$* \mathrm{E} . \mathrm{c}$ & & . & & & & & & & & & 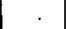 & & 1 & 1 & 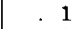 \\
\hline$* A . \operatorname{ch}$ & . & . & 2 & 2 & 5 & 2 & 30 & 4 & 3 & 2 & . & . & v & . & 40 \\
\hline *M. C & & & & & & & & & 1 & 1 & & & 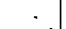 & & 1 \\
\hline *P. d & 6 & 3 & 14 & 4 & 26 & 4 & 43 & 7 & 7 & 3 & 2 & 2 & 4 & 4 & 102 \\
\hline *P. va & 52 & 7 & 43 & 8 & 85 & 15 & 142 & 10 & 36 & 10 & 38 & 11 & 149 & 6 & 545 \\
\hline B. m & 2314 & 20 & 1351 & 20 & 1684 & 20 & 1148 & 19 & 1215 & 20 & 2102 & 20 & 636 & 10 & 10450 \\
\hline B. $z$ & 84 & 16 & 119 & 16 & 45 & 14 & 63 & 16 & 102 & 20 & 169 & 19 & 54 & 10 & 636 \\
\hline B. & 1 & 1 & & & & & & & & & & & & & 1 \\
\hline B. $t$ & 6 & 4 & 3 & 3 & 7 & 2 & 4 & 4 & 10 & 5 & 9 & 6 & 1 & 1 & 40 \\
\hline L.e & 344 & 18 & 387 & 20 & 300 & 19 & 244 & 19 & 270 & 19 & 626 & 18 & 107 & 10 & 2278 \\
\hline A.C & 160 & 18 & 225 & 18 & 172 & 14 & 74 & 15 & 65 & 16 & 153 & 19 & 65 & 9 & 914 \\
\hline P. .6 & & & 2 & 1 & 4 & 3 & 1 & 1 & 3 & 3 & . & 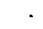 & . & . & 10 \\
\hline E. $r$ & . & . & - & . & . & & 1 & 1 &. & . & $\cdot$ & & . & - & . 1 \\
\hline M.v & . & . & . & . & & & 1 & 1 & & & 1 & 1 & . & . & 2 \\
\hline M. pe & & & & & 15 & 2 & 136 & 2 & 2 & 1 & 5 & 3 & . & . & 158 \\
\hline M. Po & 2 & 2 & 1 & 1 & 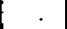 & & 1 & 1 & . & . & 4 & 3 & . & . & 8 \\
\hline P.vi & 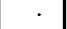 & - & 1 & 1 & . & . & & - & . & - & $\cdot$ & - & . & . & 1 \\
\hline Total & 520 & & 399 & & 463 & & 442 & & 352 & & 537 & & 261 & & 29777 \\
\hline
\end{tabular}


espèces est indépendant peut être rejetée de façon très significative (coefficient de concordance de rang de Kendall, $W=0,914$ et $X^{2}=57,58$ avec $X^{2} 0,001$ $=27,88$ pour $9 \mathrm{dd}$ ).

De la même façon, l'hypothèse nulle que le rang de ces espèces, classées selon leur fréquence, est indépendant peut être rejetée de façon très significative (coefficient de concordance de rang de Kendall, $W=0,916$ et $X^{2}=57,70$ avec $X^{2} 0,001=27,88$ pour 9 ddl).

En outre, fréquence et dominance sont très significativement ou significativement corrélées dans chaque série d'échantillons : le coefficient de corrélation de rang de Spearman, rs, présente, successivement, les valeurs suivantes : $0,866-0,851-0,857$ $0,847-0,766 \cdot 0,798$ et 0,619 (rs $0,01=0,746$, rs $0,05=0,564$ pour $\mathrm{n}=10$ ).

Ainsi les différentes séries de prélèvements sont caractérisées par leur homogénéité quant à la dominance et la fréquence de leurs formes les plus abondantes. Le rang des espèces, selon ces deux critères, n'évolue pas au cours des saisons.
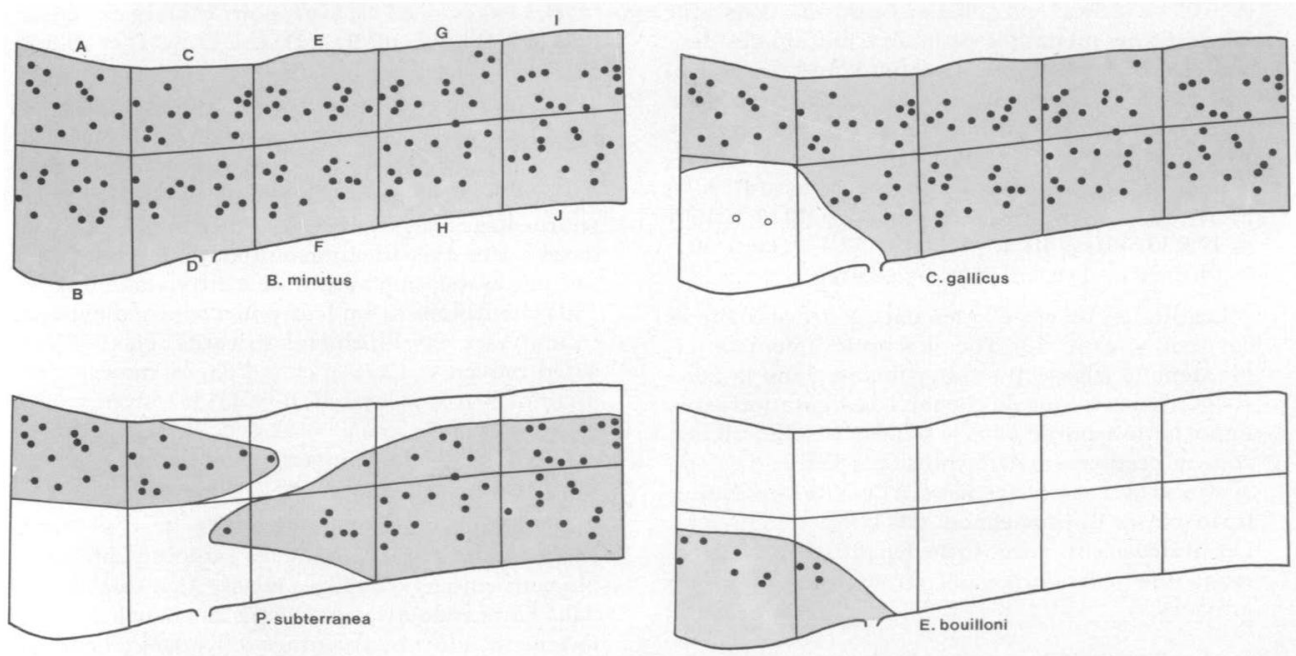

Fig. 9. Répartition des Harpacticides. Les zones colonisées sont en grisé ; chaque point correspond à un prélèvement positif 


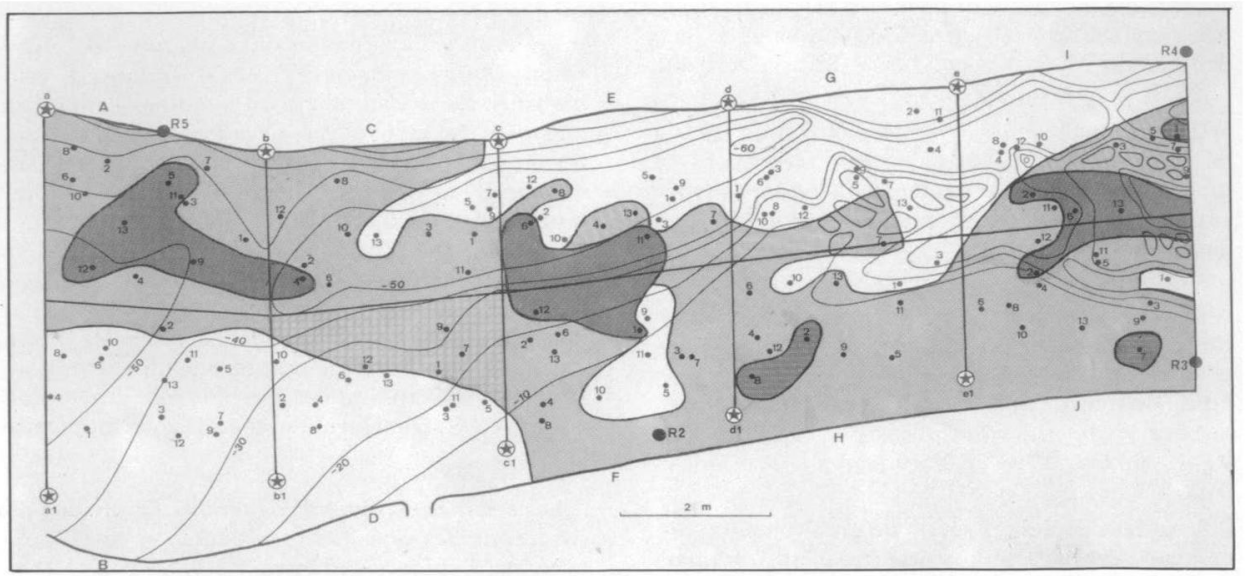

Fig. 10. Distribution des densités d'Harpacticides. Zone gris foncé : densités superieures à 42 íl. Zone gris clair : densités comprises entre 18 et 40 i/l. Zone claire: densités inférieures à $17 \mathrm{i} / \mathrm{l}$.

$X^{2} 0,05=12,59$ avec 6 ddl). Dans ces conditions, afin d'évaluer les modalités de la distribution des densités, les valeurs de celle-ci ont fait l'objet d'une classification automatique à 3 classes (agrégation autour de centres mobiles) qui conduit aux regroupements suivants : classe $\mathrm{I}, \mathrm{n}=59, \overline{\mathrm{d}}=8,5 \mathrm{i} / \mathrm{l}, \mathrm{cv}=58,6 \%$, $\mathrm{d} \operatorname{maxi}=17,1 \mathrm{i} / \mathrm{l}, \mathrm{d} \operatorname{mini}=0,3 \mathrm{i} / 1$; classe $\mathrm{II}, \mathrm{n}=$ $53, \overline{\mathrm{d}}=27,3 \mathrm{i} / \mathrm{l}, \mathrm{cv}=23,1 \%, \mathrm{~d}$ maxi $=40 \mathrm{i} / \mathrm{l}$, d mini $=17,9 \mathrm{i} / 1 ;$ classe $1 \mathrm{II}, \mathrm{n}=18, \overline{\mathrm{d}}=57,3 \mathrm{i} / 1, \mathrm{cv}=30,5$ $\%$, d maxi $=119,4, \mathrm{~d} \operatorname{mini}=42,4 \mathrm{i} / 1$.

Les limites de ces classes ont été tracées sur la carte du site (fig. 10). Tous les prélèvements à faible densité (classe 1) se distribuent dans la zone réductrice et le long du chenal. Une digitation assez importante apparaît dans le secteur $\mathrm{H}$ : elle suit une zone de creusement déterminée par le flux d'eau de rive gauche. Les prélèvements à densité moyenne et forte (classe II) proviennent des bancs de graviers. Les prélèvements à très forte den sité (classe III) forment une série de noyaux au sein des bancs de graviers.

\subsubsection{LE POURCENTAGE DES FORMES HYPOGEES}

Le pourcentage des formes hypogées dans les 130 prélèvements est, lui aussi, très variable (\% moyen
$=44,3 \%-\mathrm{cv}=63 \%$ ) avec, pour valeurs extrêmes, $0 \%(\mathrm{~B} 1, \mathrm{G} 4, \mathrm{E} 5, \mathrm{I} 8, \mathrm{~B} 12, \mathrm{D} 11, \mathrm{E} 12, \mathrm{G} 11)$ et $96,3 \%$ (C12).

Aucune variation saisonnière significative n'affecte les 7 séries de prélèvements (test de KruskalWallis : $\mathrm{H}=1 \mathrm{1}, 81$ pour $\mathrm{X}^{2} 0,05=12,59$ avec $6 \mathrm{dd}$ ). Comme pour les densités, il a été recherché si les pourcentages des hypogés sont ou non distribués au hasard. Une classification automatique à trois classes (par agrégation autour de centres mobiles) des 130 échantillons selon leur pourcentage d'hypogés conduit aux regroupements suivants : classe $\mathbf{I}, \mathbf{n}=$ $47, \%$ moyen $=12,7 \%, \mathrm{cv}=76,6 \%, \operatorname{maxi}=29,9$ $\%$. $\operatorname{mini}=0 \%$; classe $\Pi, \mathrm{n}=43, \%$ moyen $=48.8$ $\%, \mathrm{cv}=17,8 \%$, maxi $=62,4 \%$, mini $=32,4 \%$; classe III, $n=40, \%$ moyen $=76,5 \%, c v=12,5 \%$, maxi $=96,3 \%$, mini $=63,7 \%$.

Les limites de ces classes ont été tracées sur la carte du site (fig. 11). Tous les prélèvements à faible pourcentage d'hypogés (classe 1) se distribuent dans l'aire réductrice et le long du chenal. Les prélèvements à fort pourcentage d'hypogés (classe III) forment deux grands ensembles au coeur des bancs de gravier des rives droite et gauche. Les prélèvements à pourcentage moyen d'hypogés (classe II) 


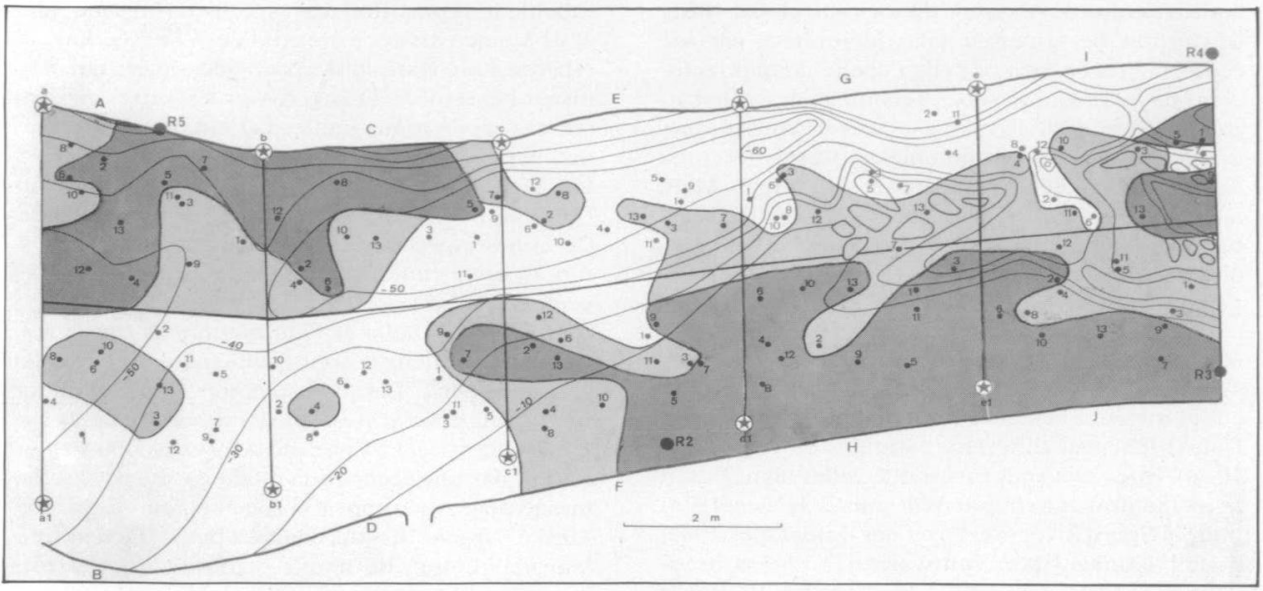

Fig- 11. Distribution des purcentages d'Harpacticides hypoges. Zone gris foncé : pourcentuges supericurs à $64 \%$. Zunc gris clair : puurcentages compris entre 32 et $62 \%$. Zone claire : pourcentages inférieurs à $30 \%$.

sont répartis à la périphérie des bancs de graviers et occupent une zone intermédiaire entre les aires des classes I et III. Les pourcentages moyens d'hypogés observés au centre de la zone réductrice sont dus a la présence d'E. botilloni espèce limitée à ce secteur.

\section{Discussion}

Ce travail avait pour but l'étude de la microdistribution (sensu Danielopol 1980, Williams 1981) des Crustacés dans le sous-écoulement d'un ruisseau des Pyrénées. Une aire de $75 \mathrm{~m}^{2}$ sur le ruisseau de Lachein a été choisie à cet effet, les prélèvements étant réalisés à la profondeur uniforme de $0,60 \mathrm{~m}$.

La dynamique des écoulements de surface de ce ruisseau influence très directement l'environnement physico-chimique de son sous-écoulement.

Sur le plan physique, elle détermine les zones de dépôts et de creusement, chacune étant caractérisée par des porosités et des granulométries spécifiques. Les températures du sous-écoulement du ruisseau de Lachein doivent également leur faible amplitude annuelle $\left(3,3^{\circ} \mathrm{C}\right)$ à la relative constance de la température des eaux de surface issues d'un aquifère karstique. Il s'agit là d'un caractère particulier puisque la plupart des études consacrées au milieu hyporhéique font état d'amplitudes annuelles dépas. sant $10^{\circ} \mathrm{C}$ (Angelier 1953, Picard 1962, Tilzer 1968, Mestrov et al 1983) et même $15^{\circ} \mathrm{C}$ (Husmann 1956, Williams \& Hynes 1974, Seyed-Reihani et al 1982, Danielopod 1983, Whitman \& Clark, 1984).

Sur le plan chimique, les écoulements de surface déterminent également les caractéristiques du sousécoulement comme en témoignent les teneurs en ions, toujours proches de celles des eaux de surface et dont elles suivent l'évolution saisonnière. C'est également un caractère noté par la plupart des auteurs (Schwoerbel 1961, Husmann 1971, Williams \& Hynes 1974, Poole \& Stewart 1976, Bretschko 1981, Hynes 1983, Dole 1983, Mestrov et al 1983). Whitman \& Clark (1982) ont synthétisé l'ensemble des données sur les teneurs en $\mathrm{O}_{2}$ dissous dans le milieu hyporhéique. La diversité des valeurs observées conduit ces auteurs à estimer que les teneurs en $\mathrm{O}_{2}$ dissous dépendent de l'interférence surfaceprofondeur avec de nombreux facteurs qui interviendraient : débits, vitesses, porosité, granulométrie, hétérogénéité. Sur le ruisseau de Lachein, 
la distribution de l'oxygène dissous suit globalement les limites des diverses zones délimitées par les écoulements de surface : elle dépend donc directement de la dynamique de ces écoulements. Ainsi, la zone réductrice de l'aval n'est pas caractérisée par une porosité et une granulométrie très différentes de celles mesurées $3 \mathrm{~m}$ en amont (secteur F). Mais les échanges avec les écoulements de surface sont bien meilleurs dans le secteur $F$ que dans la zone d'cau morte des secteurs $B$ et $\mathrm{D}$ (voir fig. 3 c).

Les tencurs en matière organique particulaire des sédiments du ruisseau de Lachein, comprises entre 4,4 et $13,6 \%$ sont du même ordre de grandeur que celles mises en évidence à $0,5 \mathrm{~m}$ de profondeur par Dole (1983) pour différentes stations du Rhône (4 à $10 \%$ ). Elles sont plus fortes que celles signalées, à la même profondeur, par Williams \& Hynes (1974) pour la Speed River (4-6\%) ou par Danielopol (1983) pour la station Eberschuttwasser (2-3\%) et beaucoup plus fortes que celles indiquées par Whitman \& Clark (1984) pour le Mill Creek $(0,32 \%)$.

Sur le plan biologique, le sous-écoulement du ruisseau de Lachein est caractérisé par sa richesse en Crustacés avec 50 espèces dénombrées dont $21 \mathrm{Har}$ pacticides, 13 Ostracodes, 9 Cyclopides, 7 Malacostracés. Cette richesse spécifique élevée est due, en partie, au fort contingent représenté par les espèces hypogées (22 espèces soit $44 \%$ des taxons dénombrés). Ainsi, pour les Harpacticides, 9 des 21 espèces recensées sont stygobies : dans leurs études respectives, Picand (1962), Ferrarese \& Sambugar (1976) n'en dénombrent que 2 sur 10 , Gourbault \& Lescher-Moutoué (1968), Braioni et al (1980), 3 sur 9, Tilzer (1968) 1 sur 12, Ronneberger (1975) 3 sur 20. Vranovsky (1984) 1 sur 8 et Kowarc (1987) 0 sur 11. Sur le Rhône, particulièrement bien êtudié, 18 espèces de Crustacés stygobies ont été recensées (Dole 1983), mais dans trois types d'habitats différents.

Les Crustacés du sous-écoulement du ruisseau de Lachein offrent une assez grande similarité faunistique avec ceu $x$ du système karstique du Baget dont l'aquifère est contigu. Seuls les Ostracodes et les Cyclopides hypogés dont aucune espèce n'est commune aux deux milieux font preuve d'une nette ségrégation alors que chez les Harpacticides et les Malacostracés, les espèces souterraines communes aux deux habitats sont majoritaires.
Seule la répartition des espèces d'Harpacticides et de Malacostracés a été étudiée. A l'exception des espèces dominantes d'Harpacticides épigés qui colonisent l'ensemble du site, toutes les autres espèces ont des répartitions limitées et notamment les formes hypogées. Les plus eurytopes, comme StenaselIus, Niphargus, Ingolfiella et C. gallicus, vivent surr l'ensemble du site à l'exclusion de la zone réductrice. Certaines espèces, plus limitées, comme Microcharon, $P$. subterranea et $P$. vandeli, occupent exclusivement les bancs de graviers alors que d'autres, comme Salentinella et Parasalentinella, ne se rencontrent que dans le sous-écoulement du chenal et à sa périphérie. Les plus sténotopes enfin, comme Vandelibathynella et E. bouilloni, sont limitées à la zone réductrice. La diversité de ces distributions se traduit par une occupation totale du site par les formes hypogées y compris la zone la moins oxygénée. Ainsi, l'oxygène dissous ne serait pas un facteur totalement limitant, du moins pour certaines formes hypogées, comme l'a souligné Danielopol (1976). Il apparaît, en outre, que les potentialités écologiques des espèces hypogées sont très variables. Marmonier et Dôle (1986) aboutissent à la même conclusion après l'étude des Amphipodes d'un bras mort du Rhône. Il s'agit là d'un phénomène important qui incite à pourstuivre l'étude de l'écologie des formes stygobies. Celles-ci, par la diversité de leurs habitats, les ségrégations dont elles témoignent, montrent, une fois encore, que l'adaptation au milieu souterrain ne sauraît être réduite à un stéréotype.

La caractéristique la plus étonnante du peuplement de Crustacés du sous-écoulement du ruisseau de Lachein réside dans sa stabilité. Elle apparaît au niveau de la composition globale du peuplement où les pourcentages des différents groupes ne paraissent pratiquement pas évoluer durant l'automne, l'hiver et le début du printemps. Cette constance est particulièrement nette chez les Harpacticides dont les rangs des espèces selon leur dominance et leur fréquence ne présentent pas de différences significatives selon les saisons. On retrouve cet te stabilité au niveau des densités globales comme des densités d'Harpacticides : si les valeurs de celles-ci témoignent d'une forte hétérogénéité spatiale, elles ne changent pas pour autant de façon significative au cours des saisons. Cette stabilité est-elle due à la constance de la température dont Angelier (1953, 1962) et Ruffo (1961) ont souligné le rôle dans l'évolution des biocénoses hyporhéiques? La question 
reste posée, mais ce résultat diffère incontestablement de tous ceux connus à ce jour dans le sousécoulement.

Chez les Harpacticides, densités et pourcentages d'hypogés ont une distribution qui se calque sur la topographie du site étudié. Les fortes valeurs de ces deux paramètres caractérisent les bancs de graviers, les zones les moins favorables étant le chenal et la zone réductrice. Ce résultat est très comparable aux observations concernant la répartition spatiale de la faune sur le Ritrodat (Bretschko \& Klemens 1986). celle des Ostracodes sur ce même Ritrodat (Marmonier 1984, 1985) ou encore celle des formes psammobies sur le Mill Creek (Whitman \& Clark 1984).

Ainsi, sur le ruisseau de Lachein, la corrélation évidente qui lie les densités et les pourcentages d'Harpacticides hypogés maximaux à des facteurs comme l' $\mathrm{O}_{2}$ dissous (la carte de la distribution de $\mathrm{I}^{\prime} \mathrm{O}_{2}$ dissous se superpose de façon étonnante à celle du pourcentage des Harpacticides hypogés), la porosité, la granulométrie, ne traduit, en fait que le rôle joué par la dynamique des écoulements de surface. Ce sont ces écoulements qui, en façonnant le milieu et en favorisant ou non les échanges avec la profondeur, régissent le peuplement. Dès 1962, Angelier a souligné l'importance de ce facteur synthétique (Marmonier \& Dole 1986).

\section{Remerciements}

Je tiens à remercier tous ceux qui m’ont aidé à réaliser ce travail : A. Descouens a participé aux prélèvethents sur le terrain, trié la faune et réalisé les dessins definitifs de cette note. A. Mangin pour la partie hydrologique, M. Bakalowicz et J. Daffis pour la géochimie, D. D'Hulst pour l'étude de la matière organique et la partie informatique mont très efficacement aidé au laboratoire comme sur le terrain avec $\mathbf{Y}$. Crouau et $\mathbf{R}$. Téchené. D. Danielopol m'a fait profiter de ses nombreuses connaissances sur le sujet. F. Boineau a saisi le manuscrit.

\section{Travaux cites}

Angeliet (E.). 1953. - Recherches Ecologiques et biogéographiques sur la faune des sables submergés. Arch. Zool. exp. gen. $90: 37-161$

Argelier (E.). 1962. - Remarques sur la répartition de la faune dans le milieu interstitiel hyporhéique. Zool. Anz., $168: 351-356$.

Bakalowicz (M.). 1979. - Contribution de la geochimie des eaux à la connaissance de l'aquifere karstique el de la karstification. Thèse Doct. Sciences, Univ. Paris-6: 269 p.
Bou (C.). 1968. - Faune souterraine du Sud-Ouest du Massif Central. II. Contribution a la connaissance de la faune des eaux souterraines de l'Albigeois. Ann. Spéléol., 23:441-473.

Bou (C.). 1979. - Etude de la faune interstitielle des alluvions du Tarn. Mise en place d'une station d'ètude et résultats prélimi naires. Bull. Fed. Tarn Spéléo-A rchéol. 16:117-130.

Bou (C) \& Rouch (R.). 1967. - Un nouveau champ de recherches sur la faune aquatique souterraine. C.R. Acad. Sc. Paris, 265 : 369-370.

Braioni (M.G.). Duzzin (B.), Centurioni (M.C.). Penna (G.), Negroni (L.) \& Campairli (S.). 1980. - Lambiente interstiziale ipor. reico del fiume Brenta e la sua fauna. Boll, Mus. Civ. St. Nat. Verona, $7: 235-274$.

Bretschko (G.). 1981. - Vertical distribution of zoobenthos in an alpine brook of the Ritrodat-Lunz study area. Verh. Internat. Vereint. Limnol, 21 : 873.876.

Bretschko (G.). 1985. - Quantitative sampling of the fauna of the gravel streams (Projek! Ritrodat-Lunz). Verh. Intemat. Verein. Limnol., 22 : 2049-2052.

Bretschko (G.) \& Klemens (W.E.) 1986. - Quantitative methods and aspects in the study of the interstitial faura of running waters. Stygologia, $2: 297-316$.

Chappuis (P.A.). 1942. - Eine neue Methode aur Untersuchung der Grundwasserfauna. Acta Sci. Math et Natur., 6 : 3-7.

Danielopol (D.). 1976. - The distribution of the fauna in the interstitial habitats of riverine sediments of the Danube and the Priesting (Austria). Int. J. Speleol., $8: 23.51$.

Danielopol (D.). 1980. - The role of the limnologists in ground water studies. Imt. Revite ges- Hydrobiol., 65 : $777-791$.

Danielopol (D.). 1982. - Phreatobiology reconsidered. Pol Arch. Hydrobiol, $29: 375-386$.

Danielopol (D.) 1983. - Der Einfluss organischer Verschmutzung. auf das Grundivasser- Ökosys lem der Donau im Raum Wien and Niederosterreich. Bundesministerium f. Gesundheit $u$. Umweltschutz, Wien, Forschungsberichte, 5: 5-160.

Danielopol (D.). Ginner (R.) \& Waidbacher (H.). 1980. - Some comments on the freezing core method of Stocker and Williams (1972). Srygo News, 3, 1:4-5.

Dole (M.J.). 1983. - Le domaine aquatique souterrain de la plaine alluviale du Rhône à l'est de Lyon ; écologie des niveaux supé. rieurs de la nappe. Thèse de $3^{*}$ cycle. Université Claude Bernard, Lyon I: $168 \mathrm{p}$.

Exner (K.K.) \& Davies (R.W.). 1979. - Comments on the use of a standpipe corer in fluvial gravels. Freshwat. Biol., $9: 77-78$.

Ferrarese (U.) \& Sambugar (B.). 1976. - Ricerche sulla fauna interstiziale iporreica dell' Adige in relazione allo stato di inquinamento del fiume. Riv. Idrobiol, $15: 47-127$.

Gibert (J.), Ginet (R.), Mathieu (J.), Reygrobellet (J.L.) \& Seyed Reihani (A.). 1977. - Structure et fonctionnement des êcosystèmes du Haut-Rhóne français. IV. Le peuplement des eaux phréatiques ; premiet's résultats. Anthls Limmed., $13: 83.97$.

Gibert (J.), Ginet (R.), Mathieu (J.) \& Reygrobellet (J.L.). 1981. Structurc et fonctionnement des écosystèmes du Haut-Rhône français. VIII. Analyse des peuplements de deux stations phréatiques alimentant des bras morts. Imt. J. Speleol. $11: 141-158$.

Godbout (L.) \& Hynes (H.B.N.), 1982. - The three dimensional dis tribution of the fauna in a single riffle in a stream in Ontario. Hydrobiologia, $97: 87.96$.

Gourbauls (N.) \& Lescher-Moutoué (F.). 1968. - Etude de la faune hypogee peuplant le sous-écoulement du Nert. Ann. Spéléol., 23 : $735-742$.

Husmann (S). 1956. - Untersuchungen über die Grundwasser. fauna zwischen Harz and Weser, Arch. Hydrobiol, $52 ; 1-184$.

Husmann (S.). 1971. - Eine Rammpumpe zur Untersuchung des subaquatischen Stygorheats. Gew. u. Abw., 50. $51: 115.119$.

Hynes (H.B.N.). 1983. - Groundwater and st ream ecology. Hydrobiologia, 100 : 93-99. 
Kirchengast (M). 1984, - Faunistische Untersuchungen im hyporheischen Interstitial des Flusses Mur (Steiermark, Osterreich). lit: Rev'ue ges. Hydrobiol, 69 : 729-746.

Kowarc (V.). 1987. - Abundanzbestimmung und populationsdynarnik der Hapacticiden (Copepoda, Crustacea) inn oberen Seebach. Jber. Biol. Stn Ltuz, $10: 53.82$.

Mangin (A.). 1974 - Contribution à l'étude hydrodynamique des aquifères karstiques. Ann. Speleol, $29: 283-332$ et 495-601

Marmonier (P.). 1984. - Vertical distribution and temporal evo lution of the ostracod assemblage of the Seebach sediments (Lunz-Austria). Jbet. Biol. Stn. Lunz, $7: 49-82$.

Maimonier (P.). 1985. - Répartition spatiale des Ostracodes dans les sédiments d'un ruisseau alpin (Le Seebach, à Lunz, Aut riche). Verh. Internat. Verein, Limnol., 22 : 2053.2057.

Marmonier (P.) \& Dole (M.J.). 1986. - Les Amphipudes des sédiments d'un bras court-circuité du Rhône. Sciences de l'eau, 5 : $461-486$.

Mest rov (M.), Stilinovic (B.), Habdija (l.), Lattinger (R.), Maloseja (Z), Kerovex (M.) \& Cicin-Sain (L.) 1983, - The ecological characteristics of interstitial underground waters in relation to the water of the river Sava. Acra Biologica, 9/1 : 5-33.

Meybeck (M.). 1984. - Les fleuves et le cycle géochimique des éléments. These Doct. Sciences, Univ. Paris-6: 506 p.

Orghidan (T.). 1959. - Ein neuer Lebensraurn des unterirdischen Wassers: der hyporheische Biotop. Arch. Hydrobiol, 55 ; $392-414$

Picard (J.Y.). 1962. - Contribution à la connaissance de la faune psammique de Lorraine, Vie et Milieu, 13:471.505.

Poole (W.C.) \& Stewart (K.W.). 1976. - The vertical distribution of macrobenthos within the Brazos River, Texas. Hydrobiolo gia, $50: 151-160$.

Ronneberger (D.). 1975. - Zur Kenntniss der Grundwasserfauna des Saale-Einzugsgebietes (Thüringen). Limnologica, 9: $323-419$.

Rouch (R.). 1968. - Cont ribution à la connaissance des Harpacticides hypogés (Crustacés Copépodes). Ann Spéléol, 23 : 5-165.

Rouch (R.). 1977. - Considérations sur l'écosystème karstique. C.R. Acad. Sc. Paris, $284: 1101-1103$.

Rouch (R.). 1984. - Les structures de peuplement des Harpacti. cides dans l'écosysteme karstique. Crustaceana, suppl. 7 360-368.
Ruffo (S.). 1961. - Prọblemi relativi al lo studio della [auna interstiziale iporreica. Boll. Zuol., $28: 273-319$.

Ruttrer-Kolisko (A.). - Biotop und Biozönose des Sandufers einiger österteichis ther Flissse. Veth. Ittemat. Verein. Limnol, 14 : 362.368.

Schwoerbel (J.). 1959. - Okologische und Tiergeographische Untersuchungen über die Milben (Acari. Hydrachnellae) der Quellen und Bäche des südlichen Schwarzwaldes und seiner Randgebiete. Arch. Hydrobiol., suppl., 24 : 385-546.

Schwoerbel (J.) 1961. - Uber die Lebensbedingungen und die Besiedlung des hyporheischen Lebensraumes. Arch. Hyarobiol. Suppl., $25: 182-214$

Seyed-Reihani (A.), Gibert (J.) \& Ginet (R.). 1982. - Structure et fonctjonmement des ćcosystemes du Haut-Rhône français. 28 : Ecologje de deux stations interstitielles; influence de la pluviositế sur leur peuplement. Pol. Arch. Hydrobiol., 29 : 501-511.

Staniord (J.A.) \& Ward (J.V.). 1988. - The hyporhe ic habitat o' river ecosystems. Nature, $335, N^{\circ} 6185: 64-66$.

Sterba (O.)\& Holzer (M.). 1977. - Fauna der interstitiellen Gewässer der Sandkiessedimente unter der aktiven Strömung. Vest. Cs. spol. zool., 41 : 144159.

Stucker (Z.S.J.) \& Williams (D.D.). 1972. - A freezing core method for describing the vertical distribution of sediments in a stream bed. Limnol. Oceanogr., 17:136-139.

Tilzer (M.). 1968. - Zur Okologie und Besiedlung des hochalpinen hyporheischen Interstitials in Arlberggebjcl (Osterreich). Arch. Hydrobiol, 65 : 253-308.

Vranovsky (M.). 1984. - Die Rheosestonmesofaura des Flusses Bela. Prace Lab. Rybar. Hydrobiol., 4 : 231.272.

Williams (D.D.) 1981. - Migrations and distributions of stream benthos. Pe rspectives in running water ecology, M.A. Lock and D.D. Williams ed, : 155-207.

Wil]iarns (D.D.) \& Hynes (H.B.N.J. 1974, - The occurence of benthos deep in the substratum of a stream. Freshwat. Biol., 4 : 233-256.

Whitman (R.L.) \& Clark (W.J.). 1982. - Availability of dissolved oxygen in interstitial waters of a sandy creek. Hydrobiologia. $92: 651-658$

Whitman (R.L.) \& Clark (W.J.). 1984. - Ecological studies of the sand-dwelling community of an East-Texas st ream. Freshwat. Invertebr. Biol., 5 : 59.79 . 